STOCHASTIC MODELING AND CONTROL

BANACH CENTER PUBLICATIONS, VOLUME 122

INSTITUTE OF MATHEMATICS

POLISH ACADEMY OF SCIENCES

WARSZAWA 2020

\title{
ASYMPTOTIC BEHAVIOR OF THE CROSS-DEPENDENCE MEASURES FOR BIDIMENSIONAL AR(1) MODEL WITH $\alpha$-STABLE NOISE
}

\author{
ALEKSANDRA GRZESIEK and AGNIESZKA WYŁOMAŃSKA \\ Faculty of Pure and Applied Mathematics, Hugo Steinhaus Center \\ Wrockaw University of Science and Technology \\ Wybrzeże Wyspiańskiego 27, 50-370 Wrocław, Poland \\ ORCID: 0000-0003-0475-964X, 0000-0001-9750-1351 \\ E-mail: aleksandra.grzesiek@pwr.edu.pl,agnieszka.wylomanska@pwr.edu.pl
}

\begin{abstract}
In this paper, we consider a bidimensional autoregressive model of order 1 with $\alpha$-stable noise. Since in this case the classical measure of dependence known as the covariance function is not defined, the spatio-temporal dependence structure is described using the alternative measures, namely the codifference and the covariation functions. Here, we investigate the asymptotic relation between these two dependence measures applied to the description of the cross-dependence of the bidimensional model. We demonstrate the case when the dependence measures are asymptotically proportional with the coefficient of proportionality equal to the parameter $\alpha$. The theoretical results are supported by illustrating the asymptotic behavior of the dependence measures for two exemplary bidimensional $\alpha$-stable $\operatorname{AR}(1)$ systems.
\end{abstract}

1. Introduction. A lot of research has shown that the models based on the heavy-tailed distributions are suitable for describing various kinds of phenomena where the assumption about the Gaussian distribution is not reasonable, see [19, 25, 2, 17, 14, 8, 30, 11, 20]. Due to the generalized central limit theorem, the natural extension of the Gaussian distribution is the $\alpha$-stable one [31, 39, 26]. The $\alpha$-stable distribution (called also stable distribution) belongs to the heavy-tailed class of distributions (except the Gaussian case of $\alpha=2$ ) and it is characterized by infinite variance. In the literature, one can find many applications of stochastic processes and time series based on the $\alpha$-stable distribution in both one-dimensional and multidimensional case, see [18, 13, 33, 37, 15, 24, 22, 38. In

2010 Mathematics Subject Classification: Primary 62M10; Secondary 37M10.

Key words and phrases: codifference, covariation, AR model, bidimensional, asymptotic behavior. The paper is in final form and no version of it will be published elsewhere. 
the multidimensional case, the classical examples are the financial data (which mostly are non-Gaussian) related to different assets (like exchange rates and metal prices) which are in some sense connected however their relationship is observed with the time shift [11, 3, 12.

However, the classical dependence measure known as the covariance function is not defined for $\alpha$-stable random vectors and thus it cannot be used to describe the structure of dependence for the $\alpha$-stable distribution-based processes. Therefore, alternative dependence measures are applied to such models, like for example covariation (and autocovariation) [31, 24, 6], codifference (and auto-codifference) [31, 24, 29, 27, 36] or fractional lower order covariance [4, 16, 32], see also [5]. The mentioned measures can be also used to describe the spatio-temporal dependence structure for multidimensional models. In the authors' previous works the cross-codifference and the cross-covariation functions were introduced for the bidimensional autoregressive model of order 1 with $\alpha$-stable noise (bidimensional $\alpha$-stable $\operatorname{AR}(1)$ model) to describe the dependence between the components of two-dimensional model, see [10, 9].

In the literature, one can find the research papers where the asymptotic behavior of the dependence measures is studied. In particular, the asymptotic relation between the auto-codifference and the auto-covariation functions of a given $\alpha$-stable distributionbased process is examined, see for example [24, 23, 21]. In this paper, we continue this research by studying the asymptotic relationship between the cross-codifference and the cross-covariation functions for the bidimensional $\alpha$-stable autoregressive model of order 1 . The work can be seen as an extension of the results presented in [21], where the asymptotic behavior of the ratio of the auto-codifference function and the auto-covariation function for one-dimensional autoregressive time series was investigated. Here we extend the approach to the two-dimensional model by studying the asymptotic relation of the cross-dependence measures describing the relationship between the components of the bidimensional model. We demonstrate the case when the dependence measures are asymptotically proportional with the coefficient of proportionality equal to the parameter $\alpha$. This result can be a starting point for the introduction of a new estimation method of the stability index in the considered model.

The paper is organized as follows. In Section 2 we present the definition of the bidimensional autoregressive model of order 1 with the $\alpha$-stable noise. In Section 3 we recall the definitions and properties of the codifference and the covariation functions together with the formulas for the cross-dependence measures corresponding to the bidimensional $\alpha$-stable AR(1) model. Section 4 contains the theorem concerning the asymptotic behavior of the ratio of the dependence measures for the bidimensional AR(1) model with the $\alpha$-stable noise. In Section 5 we illustrate the theoretical results by considering two exemplary bidimensional $\alpha$-stable AR(1) systems. In Section 6 we conclude the paper.

2. Bidimensional AR(1) model with $\alpha$-stable noise. In this section, we introduce the bidimensional autoregressive model of order 1 with $\alpha$-stable noise. Let us begin by recalling the definition of the symmetric $\alpha$-stable random vectors which are the multidimensional version of the symmetric $\alpha$-stable random variables. We remind that the 
$\alpha$-stable distribution with $0<\alpha \leq 2$ can be considered as the extension of the Gaussian distribution (the case of $\alpha=2$ ). Let $S_{d}=\{\mathbf{s}:\|\mathbf{s}\|=1\}$ be a unit sphere in $\mathbb{R}^{d}$. Then a symmetric $\alpha$-stable random vector can be presented via the characteristic function.

THEOREM 2.1 ([31]). The vector $\mathbf{Z}=\left(Z_{1}, Z_{2}, \ldots, Z_{d}\right)$ is a symmetric $\alpha$-stable vector in $\mathbb{R}^{d}$ with $0<\alpha<2$ if and only if there exists a unique symmetric finite spectral measure $\Gamma(\cdot)$ on the unit sphere $S_{d}$ such that

$$
\phi_{\mathbf{Z}}(\boldsymbol{\theta})=\mathrm{E}[\exp \{i\langle\boldsymbol{\theta}, \mathbf{Z}\rangle\}]=\exp \left\{-\int_{S_{d}}|\langle\boldsymbol{\theta}, \mathbf{s}\rangle|^{\alpha} \Gamma(d s)\right\},
$$

where $\langle\cdot, \cdot\rangle$ is the inner product.

As one can see the information about the distribution is included in the spectral measure $\Gamma(\cdot)$ and the parameter $\alpha$, also called the stability index. They fully describe $d$-dimensional symmetric $\alpha$-stable distribution which is denoted as

$$
\mathbf{Z} \sim S_{\alpha}(\Gamma)
$$

For more information about the multidimensional $\alpha$-stable distribution see for instance [31. The following definition applies to the bidimensional AR(1) time series based on the $\alpha$-stable random vector.

Definition 2.2. The time series $\{\mathbf{X}(t)\}=\left\{\left(X_{1}(t), X_{2}(t)\right)\right\}$ is a bidimensional autoregressive model of order 1 with $\alpha$-stable noise if for every $t \in \mathbb{Z}$ it satisfies

$$
\mathbf{X}(t)-\Theta \mathbf{X}(t-1)=\mathbf{Z}(t),
$$

where $\Theta$ is a $2 \times 2$ coefficient matrix given by

$$
\Theta=\left[\begin{array}{ll}
a_{1} & a_{2} \\
a_{3} & a_{4}
\end{array}\right],
$$

$\mathbf{Z}(t)$ is independent from $\mathbf{Z}(t+h)$ for all $h \neq 0$ and $\{\mathbf{Z}(t)\}=\left\{\left(Z_{1}(t), Z_{2}(t)\right)\right\}$ is a bidimensional symmetric $\alpha$-stable vector in $\mathbb{R}^{2}$ with the characteristic function defined in (1).

Moreover, we assume that for the system given by (3) the following condition is satisfied

$$
\operatorname{det}(I-z \Theta) \neq 0 \quad \text { for all } z \in \mathbb{C} \text { such that }|z| \leq 1 \text {, }
$$

i.e. the eigenvalues of the matrix $\Theta$ are less than 1 in the absolute value. Under this assumption, for each $t$ we can express $\mathbf{X}(t)$ in the causal representation as

$$
\mathbf{X}(t)=\sum_{j=0}^{+\infty} \Theta^{j} \mathbf{Z}(t-j)
$$

where the coefficients $\Theta^{j}$ are absolutely summable. In this case, $\mathbf{X}(t)$ is bounded (in the sense of the so-called covariation norm in the space of the $\alpha$-stable random variables, [31]). In this paper, we consider only the case when the eigenvalues of the matrix $\Theta$ are real numbers. 
Moreover, in [35] it was shown that for the $2 \times 2$ matrix the coefficients of $\Theta^{j}$ can be expressed as

$$
\Theta^{j}=\left[\begin{array}{cc}
\frac{\lambda_{2} \lambda_{1}^{j}-\lambda_{1} \lambda_{2}^{j}}{\lambda_{2}-\lambda_{1}}+\frac{\lambda_{2}^{j}-\lambda_{1}^{j}}{\lambda_{2}-\lambda_{1}} a_{1} & \frac{\lambda_{2}^{j}-\lambda_{1}^{j}}{\lambda_{2}-\lambda_{1}} a_{2} \\
\frac{\lambda_{2}^{j}-\lambda_{1}^{j}}{\lambda_{2}-\lambda_{1}} a_{3} & \frac{\lambda_{2} \lambda_{1}^{j}-\lambda_{1} \lambda_{2}^{j}}{\lambda_{2}-\lambda_{1}}+\frac{\lambda_{2}^{j}-\lambda_{1}^{j}}{\lambda_{2}-\lambda_{1}} a_{4}
\end{array}\right],
$$

where $\lambda_{1}, \lambda_{2}$ are two different eigenvalues of the matrix $\Theta$, i.e. when $\left(a_{1}-a_{4}\right)^{2}>-4 a_{2} a_{3}$ (and $\left|\lambda_{1}\right|<1,\left|\lambda_{2}\right|<1$ ) or

$$
\Theta^{j}=\left[\begin{array}{cc}
j \lambda^{j-1} a_{1}-(j-1) \lambda^{j} & j \lambda^{j-1} a_{2} \\
j \lambda^{j-1} a_{3} & j \lambda^{j-1} a_{4}-(j-1) \lambda^{j}
\end{array}\right],
$$

where the eigenvalues of the matrix $\Theta$ are equal $\lambda_{1}=\lambda_{2}=\lambda$, i.e. when $\left(a_{1}-a_{4}\right)^{2}=$ $-4 a_{2} a_{3}$ (and $\left.|\lambda|<1\right)$.

\section{Measures of dependence for bidimensional $\operatorname{AR}(1)$ model with $\alpha$-stable} noise. In the case of the $\alpha$-stable random vectors (for $\alpha<2$ ), the classical dependence measure known as the covariance function is not defined due to the infinite second moment and therefore other measures of dependence have to be used. The most popular ones are the codifference and the covariation given in Definition 3.1 and Definition 3.2 respectively.

Definition 3.1 ([31, 36]). Let us consider the random vector $\left(Z_{1}, Z_{2}\right)$. Then the codifference between $Z_{1}$ and $Z_{2}$ is given by

$$
\mathrm{CD}\left(Z_{1}, Z_{2}\right)=\log \mathrm{E} \exp \left\{i\left(Z_{1}-Z_{2}\right)\right\}-\log \mathrm{E} \exp \left\{i Z_{1}\right\}-\log \mathrm{E} \exp \left\{-i Z_{2}\right\} .
$$

DEFinition $3.2([31])$. Let us consider the bidimensional symmetric $\alpha$-stable random vector $\left(Z_{1}, Z_{2}\right)$ with $1<\alpha<2$ and let $\Gamma(\cdot)$ be the spectral measure of $\left(Z_{1}, Z_{2}\right)$. The covariation of $Z_{1}$ on $Z_{2}$ is the real number defined as

$$
\operatorname{CV}\left(Z_{1}, Z_{2}\right)=\int_{S_{2}} s_{1} s_{2}^{\langle\alpha-1\rangle} \Gamma(d s)
$$

where $a^{\langle p\rangle}$ is called the signed power and is equal to

$$
a^{\langle p\rangle}=|a|^{p} \operatorname{sign}(a) \text {. }
$$

Let us note that the covariation is defined only for the symmetric $\alpha$-stable random vectors. Moreover, the covariation function is not symmetric in its arguments, in contrast to the codifference function which is symmetric for the symmetric random vectors. It is worth mentioning that in the case of two independent random variables $Z_{1}$ and $Z_{2}$ both measures are equal to 0 , i.e. $\mathrm{CD}\left(Z_{1}, Z_{2}\right)=\mathrm{CV}\left(Z_{1}, Z_{2}\right)=0$. Moreover, for the Gaussian random vectors $\left(Z_{1}, Z_{2}\right)$ both measures reduce to the classical covariance function, namely

$$
\operatorname{Cov}\left(Z_{1}, Z_{2}\right)=\operatorname{CD}\left(Z_{1}, Z_{2}\right)=2 \mathrm{CV}\left(Z_{1}, Z_{2}\right),
$$

where $\operatorname{Cov}\left(Z_{1}, Z_{2}\right)$ denotes the covariance function. More properties of the codifference and the covariation can be found in [31]. 
The functions defined in $(9)$ and $(10)$ can be used to describe the interdependence of a stochastic process $\{X(t)\}$ as the auto-codifference or the auto-covariation, see [21, 23, 24, 27, 28, 36. In the authors' previous papers the measures were also applied to describe the spatio-temporal dependence structure of the bidimensional $\mathrm{AR}(1)$ model $\left\{\left(X_{1}(t), X_{2}(t)\right)\right\}$ as the cross-codifference and the cross-covariation, see [10, 9].

Now, for the bidimensional $\operatorname{AR}(1)$ model with $\alpha$-stable noise defined in (6) we can rewrite the formulas for the cross-dependence measures given in 10 taking into account the expressions for the $j$-th power of the coefficient matrix $\Theta$ given in (7) or (8). The formulas for the cross-codifference and the cross-covariation are presented in Lemma 3.3 and Lemma 3.4 , respectively.

LEMma 3.3. Let $\{\mathbf{X}(t)\}=\left\{X_{1}(t), X_{2}(t)\right\}$ be the bounded solution of (3) given by (6). If $t \in \mathbb{Z}$ and $h \in \mathbb{N}_{0}=\mathbb{N} \cup\{0\}$, then

1. in the case of two different eigenvalues of the coefficient matrix $\Theta$ denoted as $\lambda_{1}$ and $\lambda_{2}$, where $\left|\lambda_{1}\right|<1,\left|\lambda_{2}\right|<1$, and $0<\alpha<2$ we have

$$
\begin{aligned}
& \mathrm{CD}\left(X_{1}(t), X_{2}(t-h)\right)=\sum_{j=0}^{+\infty} \int_{S_{2}}\left|\lambda_{2}-\lambda_{1}\right|^{-\alpha} \\
& \cdot\left(\left|\lambda_{1}^{h}\left(\lambda_{2} \lambda_{1}^{j} s_{1}-\lambda_{1}^{j} a_{1} s_{1}-\lambda_{1}^{j} a_{2} s_{2}\right)+\lambda_{2}^{h}\left(-\lambda_{1} \lambda_{2}^{j} s_{1}+\lambda_{2}^{j} a_{1} s_{1}+\lambda_{2}^{j} a_{2} s_{2}\right)\right|^{\alpha}\right. \\
&+\left|\lambda_{2}^{j} a_{3} s_{1}-\lambda_{1}^{j} a_{3} s_{1}+\lambda_{2} \lambda_{1}^{j} s_{2}-\lambda_{1} \lambda_{2}^{j} s_{2}+\lambda_{2}^{j} a_{4} s_{2}-\lambda_{1}^{j} a_{4} s_{2}\right|^{\alpha} \\
&-\mid \lambda_{2}^{j} a_{3} s_{1}-\lambda_{1}^{j} a_{3} s_{1}+\lambda_{2} \lambda_{1}^{j} s_{2}-\lambda_{1} \lambda_{2}^{j} s_{2}+\lambda_{2}^{j} a_{4} s_{2}-\lambda_{1}^{j} a_{4} s_{2} \\
&\left.+\lambda_{1}^{h}\left(-\lambda_{2} \lambda_{1}^{j} s_{1}+\lambda_{1}^{j} a_{1} s_{1}+\lambda_{1}^{j} a_{2} s_{2}\right)+\left.\lambda_{2}^{h}\left(\lambda_{1} \lambda_{2}^{j} s_{1}-\lambda_{2}^{j} a_{1} s_{1}-\lambda_{2}^{j} a_{2} s_{2}\right)\right|^{\alpha}\right) \Gamma(d s), \\
& \mathrm{CD}\left(X_{1}(t), X_{2}(t+h)\right)=\sum_{j=0}^{+\infty} \int_{S_{2}}\left|\lambda_{2}-\lambda_{1}\right|^{-\alpha} \\
& \cdot\left(\left|\lambda_{1}^{h}\left(-\lambda_{1}^{j} a_{3} s_{1}+\lambda_{2} \lambda_{1}^{j} s_{2}-\lambda_{1}^{j} a_{4} s_{2}\right)+\lambda_{2}^{h}\left(\lambda_{2}^{j} a_{3} s_{1}-\lambda_{1} \lambda_{2}^{j} s_{2}+\lambda_{2}^{j} a_{4} s_{2}\right)\right|^{\alpha}\right. \\
& \quad+\left|\lambda_{2} \lambda_{1}^{j} s_{1}-\lambda_{1} \lambda_{2}^{j} s_{1}+\lambda_{2}^{j} a_{1} s_{1}-\lambda_{1}^{j} a_{1} s_{1}+\lambda_{2}^{j} a_{2} s_{2}-\lambda_{1}^{j} a_{2} s_{2}\right|^{\alpha} \\
& \quad-\mid \lambda_{2} \lambda_{1}^{j} s_{1}-\lambda_{1} \lambda_{2}^{j} s_{1}+\lambda_{2}^{j} a_{1} s_{1}-\lambda_{1}^{j} a_{1} s_{1}+\lambda_{2}^{j} a_{2} s_{2}-\lambda_{1}^{j} a_{2} s_{2} \\
&\left.\quad+\lambda_{1}^{h}\left(\lambda_{1}^{j} a_{3} s_{1}-\lambda_{2} \lambda_{1}^{j} s_{2}+\lambda_{1}^{j} a_{4} s_{2}\right)+\left.\lambda_{2}^{h}\left(-\lambda_{2}^{j} a_{3} s_{1}+\lambda_{1} \lambda_{2}^{j} s_{2}-\lambda_{2}^{j} a_{4} s_{2}\right)\right|^{\alpha}\right) \Gamma(d s) ;
\end{aligned}
$$

2. in the case of equal eigenvalues of the coefficient matrix $\Theta$ denoted as $\lambda_{1}=\lambda_{2}=\lambda$, where $|\lambda|<1$, and $0<\alpha<2$ we have

$$
\begin{aligned}
\operatorname{CD} & \left(X_{1}(t), X_{2}(t-h)\right)=\sum_{j=0}^{+\infty} \int_{S_{2}}\left(\mid \lambda^{h}\left(j \lambda^{j-1} a_{1} s_{1}-(j-1) \lambda^{j} s_{1}+j \lambda^{j-1} a_{2} s_{2}\right)\right. \\
+ & \left.h \lambda^{h}\left(\lambda^{j-1} a_{1} s_{1}-\lambda^{j} s_{1}+\lambda^{j-1} a_{2} s_{2}\right)\right|^{\alpha} \\
+ & \left|j \lambda^{j-1} a_{3} s_{1}-(j-1) \lambda^{j} s_{2}+j \lambda^{j-1} a_{4} s_{2}\right|^{\alpha}-\mid j \lambda^{j-1} a_{3} s_{1}-(j-1) \lambda^{j} s_{2} \\
& +j \lambda^{j-1} a_{4} s_{2}-\lambda^{h}\left(j \lambda^{j-1} a_{1} s_{1}-(j-1) \lambda^{j} s_{1}+j \lambda^{j-1} a_{2} s_{2}\right) \\
& \left.-\left.h \lambda^{h}\left(\lambda^{j-1} a_{1} s_{1}-\lambda^{j} s_{1}+\lambda^{j-1} a_{2} s_{2}\right)\right|^{\alpha}\right) \Gamma(d s),
\end{aligned}
$$




$$
\begin{aligned}
& \mathrm{CD}\left(X_{1}(t), X_{2}(t+h)\right)=\sum_{j=0}^{+\infty} \int_{S_{2}}\left(\mid \lambda^{h}\left(j \lambda^{j-1} a_{3} s_{1}-(j-1) \lambda^{j} s_{2}+j \lambda^{j-1} a_{4} s_{2}\right)\right. \\
& +\left.h \lambda^{h}\left(\lambda^{j-1} a_{3} s_{1}+\lambda^{j-1} a_{4} s_{2}-\lambda^{j} s_{2}\right)\right|^{\alpha} \\
& +\left|j \lambda^{j-1} a_{1} s_{1}-(j-1) \lambda^{j} s_{1}+j \lambda^{j-1} a_{2} s_{2}\right|^{\alpha}-\mid j \lambda^{j-1} a_{1} s_{1}-(j-1) \lambda^{j} s_{1} \\
& \quad+j \lambda^{j-1} a_{2} s_{2}-\lambda^{h}\left(j \lambda^{j-1} a_{3} s_{1}-(j-1) \lambda^{j} s_{2}+j \lambda^{j-1} a_{4} s_{2}\right) \\
& \left.-\left.h \lambda^{h}\left(\lambda^{j-1} a_{3} s_{1}+\lambda^{j-1} a_{4} s_{2}-\lambda^{j} s_{2}\right)\right|^{\alpha}\right) \Gamma(d s) .
\end{aligned}
$$

Proof. The above equations follow from the formulas presented in the authors' previous paper, see [10], and the expressions for the $j$-th power of the coefficient matrix $\Theta$ given in (7) or (8).

Lemma 3.4. Let $\{\mathbf{X}(t)\}=\left\{X_{1}(t), X_{2}(t)\right\}$ be the bounded solution of (3) given by (6). Let $t \in \mathbb{Z}$ and $h \in \mathbb{N}_{0}=\mathbb{N} \cup\{0\}$, then

1. in the case of two different eigenvalues of the coefficient matrix $\Theta$ denoted as $\lambda_{1}$ and $\lambda_{2}$, where $\left|\lambda_{1}\right|<1,\left|\lambda_{2}\right|<1$, and $1<\alpha<2$ we have

$$
\begin{aligned}
& \mathrm{CV}\left(X_{1}(t), X_{2}(t-h)\right) \\
= & \sum_{j=0}^{+\infty} \int_{S_{2}}\left(\frac{\lambda_{2}^{j} a_{3} s_{1}-\lambda_{1}^{j} a_{3} s_{1}+\lambda_{2} \lambda_{1}^{j} s_{2}-\lambda_{1} \lambda_{2}^{j} s_{2}+\lambda_{2}^{j} a_{4} s_{2}-\lambda_{1}^{j} a_{4} s_{2}}{\lambda_{2}-\lambda_{1}}\right) \\
& \left(\frac{\lambda_{1}^{h}\left(\lambda_{2} \lambda_{1}^{j} s_{1}-\lambda_{1}^{j} a_{1} s_{1}-\lambda_{1}^{j} a_{2} s_{2}\right)+\lambda_{2}^{h}\left(-\lambda_{1} \lambda_{2}^{j} s_{1}+\lambda_{2}^{j} a_{1} s_{1}+\lambda_{2}^{j} a_{2} s_{2}\right)}{\lambda_{2}-\lambda_{1}}\right) \Gamma(d s), \\
\mathrm{CV} & \left(X_{1}(t), X_{2}(t+h)\right) \\
= & \sum_{j=0}^{+\infty} \int_{S_{2}}\left(\frac{\lambda_{2} \lambda_{1}^{j} s_{1}-\lambda_{1} \lambda_{2}^{j} s_{1}+\lambda_{2}^{j} a_{1} s_{1}-\lambda_{1}^{j} a_{1} s_{1}+\lambda_{2}^{j} a_{2} s_{2}-\lambda_{1}^{j} a_{2} s_{2}}{\lambda_{2}-\lambda_{1}}\right) \\
( & \left.\frac{\lambda_{1}^{h}\left(-\lambda_{1}^{j} a_{3} s_{1}+\lambda_{2} \lambda_{1}^{j} s_{2}-\lambda_{1}^{j} a_{4} s_{2}\right)+\lambda_{2}^{h}\left(\lambda_{2}^{j} a_{3} s_{1}-\lambda_{1} \lambda_{2}^{j} s_{2}+\lambda_{2}^{j} a_{4} s_{2}\right)}{\lambda_{2}-\lambda_{1}}\right)^{\langle\alpha-1\rangle} \Gamma(d s) ;
\end{aligned}
$$

2. in the case of equal eigenvalues of the coefficient matrix $\Theta$ denoted as $\lambda_{1}=\lambda_{2}=\lambda$, where $|\lambda|<1$, and $1<\alpha<2$ we have

$$
\begin{aligned}
\mathrm{CV}\left(X_{1}(t), X_{2}(t-h)\right)= & \sum_{j=0}^{+\infty} \int_{S_{2}}\left(j \lambda^{j-1} a_{3} s_{1}-(j-1) \lambda^{j} s_{2}+j \lambda^{j-1} a_{4} s_{2}\right){ }^{\langle\alpha-1\rangle} \\
\left(\lambda^{h}\left(j \lambda^{j-1} a_{1} s_{1}-(j-1) \lambda^{j} s_{1}+j \lambda^{j-1} a_{2} s_{2}\right)\right. & \\
& \left.+h \lambda^{h}\left(\lambda^{j-1} a_{1} s_{1}-\lambda^{j} s_{1}+\lambda^{j-1} a_{2} s_{2}\right)\right) \Gamma(d s), \\
\mathrm{CV}\left(X_{1}(t), X_{2}(t+h)\right)= & \sum_{j=0}^{+\infty} \int_{S_{2}}\left(j \lambda^{j-1} a_{1} s_{1}-(j-1) \lambda^{j} s_{1}+j \lambda^{j-1} a_{2} s_{2}\right) \\
& \left(\lambda^{h}\left(j \lambda^{j-1} a_{3} s_{1}-(j-1) \lambda^{j} s_{2}+j \lambda^{j-1} a_{4} s_{2}\right)\right. \\
& \left.+h \lambda^{h}\left(\lambda^{j-1} a_{3} s_{1}+\lambda^{j-1} a_{4} s_{2}-\lambda^{j} s_{2}\right)\right)^{\langle\alpha-1\rangle} \Gamma(d s) .
\end{aligned}
$$


Proof. The above equations follow from the formulas presented in [10, and the expressions for the $j$-th power of the coefficient matrix $\Theta$ given in (7) or (8).

4. The asymptotic behavior for the ratio of codifference and covariation for bidimensional $\mathbf{A R}(1)$ model with $\alpha$-stable noise. In this section, we examine the asymptotic relation of the cross-codifference function and the cross-covariation function for the bidimensional $\mathrm{AR}(1)$ model with $\alpha$-stable noise presented in Section 2 Before formulating the relevant theorem, we separately consider the asymptotic behavior of both measures for $h \rightarrow+\infty$ in Lemma 4.1 and Lemma 4.2 respectively. For both functions, we distinguish five separate cases, see Table 1.

\begin{tabular}{|c|c|c|c|c|}
\hline$\left|\lambda_{1}\right|>\left|\lambda_{2}\right|$ & $\left|\lambda_{1}\right|<\left|\lambda_{2}\right|$ & $\lambda_{1}=\lambda_{2}=\lambda$ & $\begin{array}{c}\lambda_{1}=-\lambda_{2} \\
\text { and } h \text { even }\end{array}$ & $\begin{array}{c}\lambda_{1}=-\lambda_{2} \\
\text { and } h \text { odd }\end{array}$ \\
\hline Case I & Case II & Case III & Case IV & Case V \\
\hline
\end{tabular}

Table 1. The cases considered for the asymptotic behavior of the cross-dependence measures LEMMA 4.1. If $\{\mathbf{X}(t)\}=\left\{X_{1}(t), X_{2}(t)\right\}$ for $t \in Z$ is the bounded solution of (3) given by (6) with $1<\alpha<2$, then for the cross-codifference function the following asymptotic formulas are true when $h \rightarrow+\infty$

a)

$$
\mathrm{CD}\left(X_{1}(t), X_{2}(t-h)\right) \sim \begin{cases}\alpha D_{1} \lambda_{1}^{h} & \text { Case I, } \\ \alpha D_{2} \lambda_{2}^{h} & \text { Case II, } \\ \alpha D_{3} h \lambda^{h} & \text { Case III, } \\ \alpha\left(D_{1}+D_{2}\right) \lambda_{1}^{h} & \text { Case IV, } \\ \alpha\left(D_{1}-D_{2}\right) \lambda_{1}^{h} & \text { Case V, }\end{cases}
$$

where the constants $D_{1}, D_{2}$ and $D_{3}$ are given in (31), (33) and (43), respectively.

b)

$$
\mathrm{CD}\left(X_{1}(t), X_{2}(t+h)\right) \sim \begin{cases}\alpha D_{4} \lambda_{1}^{h} & \text { Case I, } \\ \alpha D_{5} \lambda_{2}^{h} & \text { Case II, } \\ \alpha D_{6} h \lambda^{h} & \text { Case III, } \\ \alpha\left(D_{4}+D_{5}\right) \lambda_{1}^{h} & \text { Case IV, } \\ \alpha\left(D_{4}-D_{5}\right) \lambda_{1}^{h} & \text { Case V, }\end{cases}
$$

where the constants $D_{4}, D_{5}$ and $D_{6}$ are given in (47), (50) and (56), respectively.

Proof. The proof is given in Appendix A.

LEMMA 4.2. If $\{\mathbf{X}(t)\}=\left\{X_{1}(t), X_{2}(t)\right\}$ for $t \in Z$ is the bounded solution of (3) given by (6) with $1<\alpha<2$, then for the cross-covariation function we have

a) the following asymptotic formulas when $h \rightarrow+\infty$

$$
\mathrm{CV}\left(X_{1}(t), X_{2}(t-h)\right) \sim \begin{cases}D_{1} \lambda_{1}^{h} & \text { Case I, } \\ D_{2} \lambda_{2}^{h} & \text { Case II, } \\ D_{3} h \lambda^{h} & \text { Case III, }\end{cases}
$$

or the following exact formulas

$$
\mathrm{CV}\left(X_{1}(t), X_{2}(t-h)\right)= \begin{cases}\left(D_{1}+D_{2}\right) \lambda_{1}^{h} & \text { Case IV } \\ \left(D_{1}-D_{2}\right) \lambda_{1}^{h} & \text { Case V }\end{cases}
$$


where the constants $D_{1}, D_{2}$ and $D_{3}$ are given in (31), (33) and $(43)$, respectively.

b) the following asymptotic formulas when $h \rightarrow+\infty$

or the following exact formulas

$$
\mathrm{CV}\left(X_{1}(t), X_{2}(t+h)\right) \sim \begin{cases}D_{7}\left(\lambda_{1}^{h}\right)^{\langle\alpha-1\rangle} & \text { Case I, } \\ D_{8}\left(\lambda_{2}^{h}\right)^{\langle\alpha-1\rangle} & \text { Case II, } \\ D_{9}\left(h \lambda^{h}\right)^{\langle\alpha-1\rangle} & \text { Case III }\end{cases}
$$

$$
\mathrm{CV}\left(X_{1}(t), X_{2}(t+h)\right)= \begin{cases}D_{10}\left(\lambda_{1}^{h}\right)^{\langle\alpha-1\rangle} & \text { Case IV } \\ D_{11}\left(\lambda_{1}^{h}\right)^{\langle\alpha-1\rangle} & \text { Case V }\end{cases}
$$

where the constants $D_{7}, D_{8}, D_{9}, D_{10}$ and $D_{11}$ are given in (67), 69, (80), (71) and 73 , respectively.

Proof. The proof is given in Appendix B.

Theorem 4.3. If $\{\mathbf{X}(t)\}=\left\{X_{1}(t), X_{2}(t)\right\}$ is the bounded solution of (3) given by (6), then for $1<\alpha<2$ and $t \in Z$ :

a) if $\mathrm{CV}\left(X_{1}(t), X_{2}(t-h)\right) \neq 0$, then

$$
\lim _{h \rightarrow+\infty} \frac{\mathrm{CD}\left(X_{1}(t), X_{2}(t-h)\right)}{\mathrm{CV}\left(X_{1}(t), X_{2}(t-h)\right)}=\alpha,
$$

b) if $\mathrm{CV}\left(X_{1}(t), X_{2}(t+h)\right) \neq 0$, then

$$
\lim _{h \rightarrow+\infty} \frac{\mathrm{CD}\left(X_{1}(t), X_{2}(t+h)\right)}{\operatorname{CV}\left(X_{1}(t), X_{2}(t+h)\right)}=0 .
$$

Proof. The limits given in Theorem 4.3 follow directly from the formulas describing the asymptotic behavior of the cross-dependence measures given in Lemmas 4.1, 4.2 ,

5. Example. To illustrate the theoretical results showing the asymptotic behavior of the dependence measures for the bidimensional autoregressive model of order 1 with general symmetric $\alpha$-stable noise, we consider two exemplary bidimensional $\operatorname{AR}(1)$ time series with the following coefficient matrices

$$
\Theta_{1}=\left[\begin{array}{ll}
-0.2 & 0.1 \\
-0.3 & 0.6
\end{array}\right] \quad \text { and } \quad \Theta_{2}=\left[\begin{array}{cc}
0.5 & 0.1 \\
-0.1 & 0.7
\end{array}\right] \text {. }
$$

Since the eigenvalues of the matrix $\Theta_{1}\left(\lambda_{1} \approx-0.16, \lambda_{2} \approx 0.56\right)$ and $\Theta_{2}\left(\lambda_{1}=\lambda_{2}=\right.$ $\lambda=0.6$ ) are less than 1 in absolute value, for both time series there exists the bounded solution given in (6). Moreover, let us assume that the spectral measure of the symmetric $\alpha$-stable random vector is concentrated on four points on the unit sphere $S_{2}$, namely it has the following form

$$
\begin{aligned}
& \Gamma(\cdot)=0.5 \delta\left(\left(\frac{1}{2}, \frac{\sqrt{3}}{2}\right)\right)+0.5 \delta\left(\left(-\frac{1}{2},-\frac{\sqrt{3}}{2}\right)\right) \\
& +0.2 \delta\left(\left(-\frac{1}{2}, \frac{\sqrt{3}}{2}\right)\right)+0.2 \delta\left(\left(\frac{1}{2},-\frac{\sqrt{3}}{2}\right)\right) \text {. }
\end{aligned}
$$



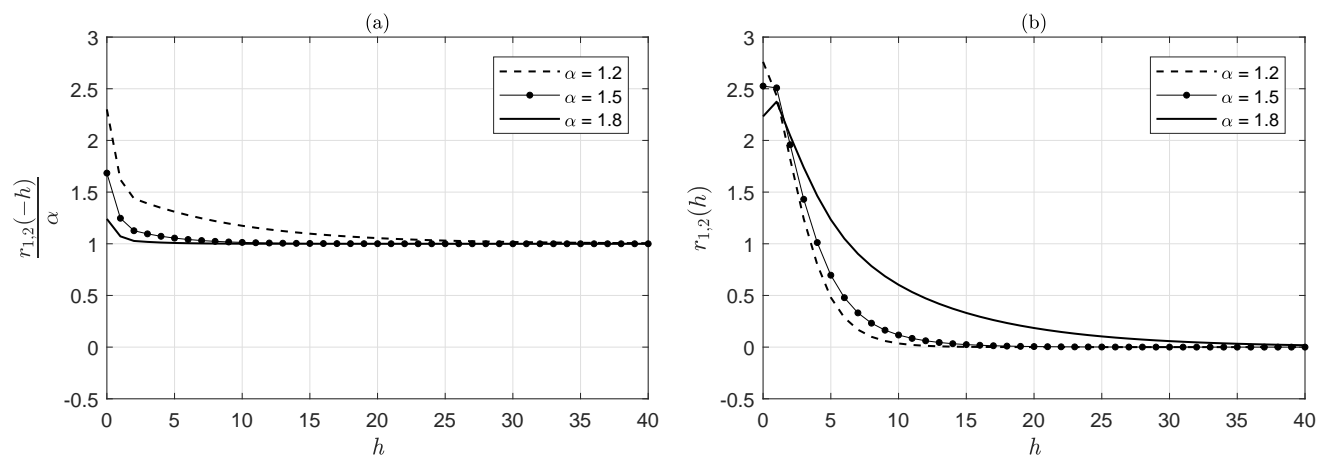

Fig. 1. The terms $\frac{r_{1,2}(-h)}{\alpha}$ (panel (a)) and $r_{1,2}(h)$ (panel (b)) presenting the asymptotic behavior of the cross-dependence measures for the bidimensional $\mathrm{AR}(1)$ model with coefficients given in matrix $\Theta_{1}$ and the $\alpha$-stable noise with the symmetric spectral measure specified in 21 for $\alpha=1.2$ (dashed line), $\alpha=1.5$ (solid line with dots) and $\alpha=1.8$ (solid line)

(a)

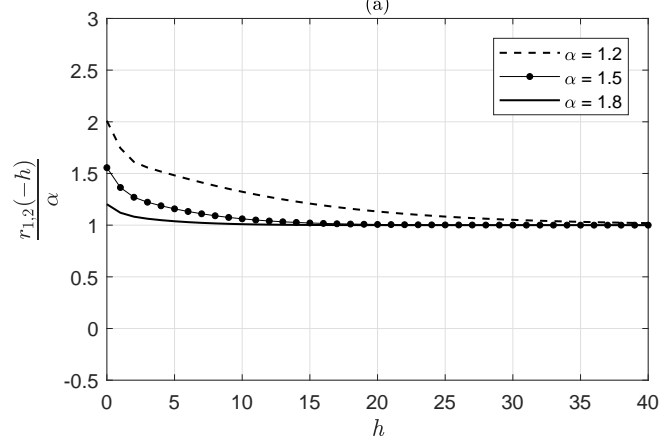

(b)

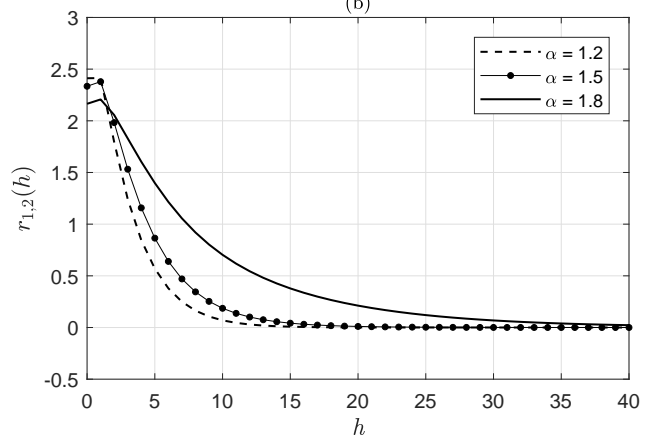

Fig. 2. The terms $\frac{r_{1,2}(-h)}{\alpha}$ (panel (a)) and $r_{1,2}(h)$ (panel (b)) presenting the asymptotic behavior of the dependence measures for the bidimensional AR(1) model with coefficients given in matrix $\Theta_{2}$ and the $\alpha$-stable noise with the symmetric spectral measure specified in 21 for $\alpha=1.2$ (dashed line), $\alpha=1.5$ (solid line with dots) and $\alpha=1.8$ (solid line)

This specific model was considered in the authors' previous paper, see Example 3.3 in [10], where the formulas for the corresponding cross-dependence measures are presented. Now, in order to demonstrate the asymptotic relation between the crosscodifference and the cross-covariation we plot the functions

$$
\frac{r_{1,2}(-h)}{\alpha}=\frac{\mathrm{CD}\left(X_{1}(t), X_{2}(t-h)\right)}{\alpha \operatorname{CV}\left(X_{1}(t), X_{2}(t-h)\right)} \quad \text { and } \quad r_{1,2}(h)=\frac{\operatorname{CD}\left(X_{1}(t), X_{2}(t+h)\right)}{\operatorname{CV}\left(X_{1}(t), X_{2}(t+h)\right)}
$$

for $h=0,1, \ldots, 40$ and $\alpha=1.2, \alpha=1.5$ and $\alpha=1.8$. The graphs are presented in Figure 1 (the model with coefficients given in matrix $\Theta_{1}$ ) and in Figure 2 (the model with coefficients given in matrix $\Theta_{2}$ ). According to the results presented in Section 4. the term $\frac{r_{1,2}(-h)}{\alpha}$ tends to 1 (panel (a) of Figures 1 2) and the term $r_{1,2}(h)$ tends to 0 (panel (b) of Figures 1 2). Moreover, we can see that the rate of the convergence depends 
on the value of the parameter $\alpha$. The quotient denoted as $r_{1,2}(-h)$ converges faster for larger values of the stability index, on the contrary to the quotient denoted as $r_{1,2}(h)$ for which the larger value the parameter $\alpha$ takes, the slower is the convergence.

6. Conclusions. In this paper, we considered a bidimensional autoregressive model of order 1 with $\alpha$-stable noise. For this process, the structure of dependence can be described using the codifference function and the covariation function. Here, we examined the asymptotic relation between these two measures applied to the description of the crossdependence between the components of the bidimensional model (the cross-codifference function and the cross-covariation function). The main results concerning the asymptotic relationship between the considered dependence measures are presented in Theorem 4.3 where we identified the case when the measures are asymptotically proportional with the coefficient of proportionality equal to the parameter $\alpha$. The conclusions obtained here constitute an extension of the results presented in [21] for the one-dimensional autoregressive models and may be useful in the context of the parameter $\alpha$ estimation. This can be a starting point for the introduction of a new estimation algorithm for the stability index for the bidimensional autoregressive model of order 1 with the $\alpha$-stable noise.

\section{Appendix A: Proof of Lemma 4.1}

a) First, we examine the asymptotic behavior of $\operatorname{CD}\left(X_{1}(t), X_{2}(t-h)\right)$ for $h \rightarrow+\infty$.

- Let us consider the case of two different real eigenvalues of the coefficient matrix $\Theta$, $\lambda_{1} \neq \lambda_{2}$, and $\left|\lambda_{1}\right|<1,\left|\lambda_{2}\right|<1$. For the cross-codifference function $\operatorname{CD}\left(X_{1}(t), X_{2}(t-h)\right)$ given in Lemma 3.3 (see [11) ) we introduce the notation

$$
\begin{aligned}
A_{1}\left(s_{1}, s_{2}, a_{1}, a_{2}, \lambda_{1}, \lambda_{2}, j\right) & =A_{1}=\frac{\lambda_{2} \lambda_{1}^{j} s_{1}-\lambda_{1}^{j} a_{1} s_{1}-\lambda_{1}^{j} a_{2} s_{2}}{\lambda_{2}-\lambda_{1}}, \\
B_{1}\left(s_{1}, s_{2}, a_{1}, a_{2}, \lambda_{1}, \lambda_{2}, j\right) & =B_{1}=\frac{-\lambda_{1} \lambda_{2}^{j} s_{1}+\lambda_{2}^{j} a_{1} s_{1}+\lambda_{2}^{j} a_{2} s_{2}}{\lambda_{2}-\lambda_{1}}, \\
C_{1}\left(s_{1}, s_{2}, a_{3}, a_{4}, \lambda_{1}, \lambda_{2}, j\right) & =C_{1} \\
& =\frac{\lambda_{1}^{j}\left(-a_{3} s_{1}+\lambda_{2} s_{2}-a_{4} s_{2}\right)+\lambda_{2}^{j}\left(a_{3} s_{1}-\lambda_{1} s_{2}+a_{4} s_{2}\right)}{\lambda_{2}-\lambda_{1}} .
\end{aligned}
$$

In the following part of the proof, the above expressions will be referred as $A_{1}, B_{1}$ and $C_{1}$ to simplify the notation. However, it is important to notice that although they do not depend on $h$, they are dependent on $j, s_{1}, s_{2}$ and the coefficients $a_{1}, a_{2}, a_{3}, a_{4}$ and thus on the eigenvalues of matrix $\Theta$. Now, the cross-codifference function $\operatorname{CD}\left(X_{1}(t), X_{2}(t-h)\right)$ given in 11 takes the form

$$
\begin{aligned}
& \operatorname{CD}\left(X_{1}(t), X_{2}(t-h)\right) \\
& \quad=\sum_{j=0}^{+\infty} \int_{S_{2}}\left(\left|\lambda_{1}^{h} A_{1}+\lambda_{2}^{h} B\right|^{\alpha}+\left|C_{1}\right|^{\alpha}-\left|C_{1}-\left(\lambda_{1}^{h} A_{1}+\lambda_{2}^{h} B_{1}\right)\right|^{\alpha}\right) \Gamma(d s) .
\end{aligned}
$$


I) Consider the case of $\left|\lambda_{1}\right|>\left|\lambda_{2}\right|$. Now, we split the proof into two parts. Namely, we show that

i) $\lim _{h \rightarrow+\infty} \sum_{j=0}^{+\infty} \int_{S_{2}} \frac{\left|\lambda_{1}^{h} A_{1}+\lambda_{2}^{h} B_{1}\right|^{\alpha}+\left|C_{1}\right|^{\alpha}-\left|C_{1}-\left(\lambda_{1}^{h} A_{1}+\lambda_{2}^{h} B_{1}\right)\right|^{\alpha}}{\lambda_{1}^{h}} \Gamma(d s)$

$$
=\sum_{j=0}^{+\infty} \lim _{h \rightarrow+\infty} \int_{S_{2}} \frac{\left|\lambda_{1}^{h} A_{1}+\lambda_{2}^{h} B_{1}\right|^{\alpha}+\left|C_{1}\right|^{\alpha}-\left|C_{1}-\left(\lambda_{1}^{h} A_{1}+\lambda_{2}^{h} B_{1}\right)\right|^{\alpha}}{\lambda_{1}^{h}} \Gamma(d s),
$$

ii) $\lim _{h \rightarrow+\infty} \int_{S_{2}} \frac{\left|\lambda_{1}^{h} A_{1}+\lambda_{2}^{h} B_{1}\right|^{\alpha}+\left|C_{1}\right|^{\alpha}-\left|C_{1}-\left(\lambda_{1}^{h} A_{1}+\lambda_{2}^{h} B_{1}\right)\right|^{\alpha}}{\lambda_{1}^{h}} \Gamma(d s)$

$$
=\int_{S_{2}} \lim _{h \rightarrow+\infty} \frac{\left|\lambda_{1}^{h} A_{1}+\lambda_{2}^{h} B_{1}\right|^{\alpha}+\left|C_{1}\right|^{\alpha}-\left|C_{1}-\left(\lambda_{1}^{h} A_{1}+\lambda_{2}^{h} B_{1}\right)\right|^{\alpha}}{\lambda_{1}^{h}} \Gamma(d s) .
$$

Let us focus on part i). According to the dominated convergence theorem [34, Ch. 5], the equality given in (24) is true if the infinite sum over $j$ converges uniformly. Let us notice that using the following inequalities true for all $n, m \in \mathbb{R}, 1<\alpha<2$ [17]

$$
\begin{aligned}
\left.|| n\right|^{\alpha}+|m|^{\alpha}-|n+m|^{\alpha} \mid & \leq(\alpha+1)|n|^{\alpha}+\alpha|n||m|^{\alpha-1} \\
|n+m|^{\alpha} & \leq 2^{\alpha-1}\left(|n|^{\alpha}+|m|^{\alpha}\right),
\end{aligned}
$$

one can show that for all $j \in \mathbb{N}$ we have

$$
\begin{aligned}
& \left|\int_{S_{2}} \frac{\left|\lambda_{1}^{h} A_{1}+\lambda_{2}^{h} B_{1}\right|^{\alpha}+\left|C_{1}\right|^{\alpha}-\left|C_{1}-\left(\lambda_{1}^{h} A_{1}+\lambda_{2}^{h} B_{1}\right)\right|^{\alpha}}{\lambda_{1}^{h}} \Gamma(d s)\right| \\
& \leq \int_{S_{2}}\left|\frac{\left|\lambda_{1}^{h} A_{1}+\lambda_{2}^{h} B_{1}\right|^{\alpha}+\left|C_{1}\right|^{\alpha}-\left|C_{1}-\left(\lambda_{1}^{h} A_{1}+\lambda_{2}^{h} B_{1}\right)\right|^{\alpha}}{\lambda_{1}^{h}}\right| \Gamma(d s) \\
& \leq \int_{S_{2}} \frac{(\alpha+1)\left|\lambda_{1}^{h} A_{1}+\lambda_{2}^{h} B_{1}\right|^{\alpha}+\alpha\left|\lambda_{1}^{h} A_{1}+\lambda_{2}^{h} B_{1}\right|\left|C_{1}\right|^{\alpha-1}}{\left|\lambda_{1}^{h}\right|} \Gamma(d s) \\
& =(\alpha+1)\left|\lambda_{1}^{h}\right|^{\alpha-1} \int_{S_{2}}\left|A_{1}+\left(\frac{\lambda_{2}}{\lambda_{1}}\right)^{h} B_{1}\right|^{\alpha} \Gamma(d s) \\
& +\alpha \int_{S_{2}}\left|A_{1}+\left(\frac{\lambda_{2}}{\lambda_{1}}\right)^{h} B_{1}\right|\left|C_{1}\right|^{\alpha-1} \Gamma(d s) \\
& \leq(\alpha+1)\left|\lambda_{1}^{h}\right|^{\alpha-1} 2^{\alpha-1} \int_{S_{2}}\left(\left|A_{1}\right|^{\alpha}+\left|\left(\frac{\lambda_{2}}{\lambda_{1}}\right)^{h} B_{1}\right|^{\alpha}\right) \Gamma(d s) \\
& +\alpha \int_{S_{2}}\left(\left|A_{1}\right|+\left|\left(\frac{\lambda_{2}}{\lambda_{1}}\right)^{h} B_{1}\right|\right)\left|C_{1}\right|^{\alpha-1} \Gamma(d s) \\
& \leq 2^{\alpha-1}(\alpha+1)\left(\int_{S_{2}}\left|A_{1}\right|^{\alpha} \Gamma(d s)+\int_{S_{2}}\left|B_{1}\right|^{\alpha} \Gamma(d s)\right) \\
& +\alpha\left(\int_{S_{2}}\left|A_{1}\right|\left|C_{1}\right|^{\alpha-1} \Gamma(d s)+\int_{S_{2}}\left|B_{1}\right|\left|C_{1}\right|^{\alpha-1} \Gamma(d s)\right)=M_{j},
\end{aligned}
$$

and $M_{j}$ is independent of $h$. Now, the infinite sum under the limit in 24) converges uniformly if the infinite sum of $M_{j}$ over $j \in \mathbb{N}$ converges, which is equivalent to the 
following set of conditions

$$
\begin{array}{ll}
\sum_{j=0}^{+\infty} \int_{S_{2}}\left|A_{1}\right|^{\alpha} \Gamma(d s)<+\infty, & \sum_{j=0}^{+\infty} \int_{S_{2}}\left|A_{1}\right|\left|C_{1}\right|^{\alpha-1} \Gamma(d s)<+\infty, \\
\sum_{j=0}^{+\infty} \int_{S_{2}}\left|B_{1}\right|^{\alpha} \Gamma(d s)<+\infty, & \sum_{j=0}^{+\infty} \int_{S_{2}}\left|B_{1}\right|\left|C_{1}\right|^{\alpha-1} \Gamma(d s)<+\infty,
\end{array}
$$

which are always satisfied (see Remark C.1 and consequently the equality given in (24) is true.

Let us now focus on part ii). Similarly, according to the dominated convergence theorem, the equality given in 25 is satisfied if the integrand is dominated by an integrable function independent of $h$. Let us notice that using the formulas given in 26 for a fixed $\mathbf{s}=\left(s_{1}, s_{2}\right) \in S_{2}$ we have

$$
\begin{aligned}
& \left|\frac{\left|\lambda_{1}^{h} A_{1}+\lambda_{2}^{h} B_{1}\right|^{\alpha}+\left|C_{1}\right|^{\alpha}-\left|C_{1}-\left(\lambda_{1}^{h} A_{1}+\lambda_{2}^{h} B_{1}\right)\right|^{\alpha}}{\lambda_{1}^{h}}\right| \\
& \leq \frac{(\alpha+1)\left|\lambda_{1}^{h} A_{1}+\lambda_{2}^{h} B_{1}\right|^{\alpha}+\alpha\left|\lambda_{1}^{h} A_{1}+\lambda_{2}^{h} B_{1}\right|\left|C_{1}\right|^{\alpha-1}}{\left|\lambda_{1}^{h}\right|} \\
& =(\alpha+1)\left|\lambda_{1}^{h}\right|^{\alpha-1}\left|A_{1}+\left(\frac{\lambda_{2}}{\lambda_{1}}\right)^{h} B_{1}\right|^{\alpha}+\alpha\left|A_{1}+\left(\frac{\lambda_{2}}{\lambda_{1}}\right)^{h} B_{1}\right|\left|C_{1}\right|^{\alpha-1} \\
& \leq(\alpha+1)\left|\lambda_{1}^{h}\right|^{\alpha-1} 2^{\alpha-1}\left(\left|A_{1}\right|^{\alpha}+\left|\left(\frac{\lambda_{2}}{\lambda_{1}}\right)^{h} B_{1}\right|^{\alpha}\right)+\alpha\left(\left|A_{1}\right|+\left|\left(\frac{\lambda_{2}}{\lambda_{1}}\right)^{h} B_{1}\right|\right)\left|C_{1}\right|^{\alpha-1} \\
& \leq 2^{\alpha-1}(\alpha+1)\left(\left|A_{1}\right|^{\alpha}+\left|B_{1}\right|^{\alpha}\right)+\alpha\left(\left|A_{1}\right|\left|C_{1}\right|^{\alpha-1}+\left|B_{1}\right|\left|C_{1}\right|^{\alpha-1}\right),
\end{aligned}
$$

which is independent of $h$. Since the dominating function should be integrable we deduce that for all $j \in \mathbb{N}$ the following set of conditions has to be satisfied:

$$
\begin{array}{ll}
\int_{S_{2}}\left|A_{1}\right|^{\alpha} \Gamma(d s)<+\infty, & \int_{S_{2}}\left|A_{1}\right|\left|C_{1}\right|^{\alpha-1} \Gamma(d s)<+\infty, \\
\int_{S_{2}}\left|B_{1}\right|^{\alpha} \Gamma(d s)<+\infty, & \int_{S_{2}}\left|B_{1}\right|\left|C_{1}\right|^{\alpha-1} \Gamma(d s)<+\infty .
\end{array}
$$

Let us notice that the above conditions are satisfied since the conditions given in (27) hold. Now, we calculate the limit of the integrand given in 25 for a fixed $\mathbf{s}=\left(s_{1}, s_{2}\right) \in S_{2}$, namely

$$
\lim _{h \rightarrow+\infty} \frac{\left|\lambda_{1}^{h} A_{1}+\lambda_{2}^{h} B_{1}\right|^{\alpha}+\left|C_{1}\right|^{\alpha}-\left|C_{1}-\left(\lambda_{1}^{h} A_{1}+\lambda_{2}^{h} B_{1}\right)\right|^{\alpha}}{\lambda_{1}^{h}} .
$$

Let us notice that for $h \rightarrow+\infty$

$$
\begin{aligned}
& \left|\lambda_{1}^{h} A_{1}+\lambda_{2}^{h} B_{1}\right|^{\alpha}+\left|C_{1}\right|^{\alpha}-\left|C_{1}-\left(\lambda_{1}^{h} A_{1}+\lambda_{2}^{h} B_{1}\right)\right|^{\alpha} \\
& =\left|\lambda_{1}^{h}\left(A_{1}+\left(\lambda_{2} / \lambda_{1}\right)^{h} B_{1}\right)\right|^{\alpha}+\left|C_{1}\right|^{\alpha}-\left|C_{1}-\lambda_{1}^{h}\left(A_{1}+\left(\lambda_{2} / \lambda_{1}\right)^{h} B_{1}\right)\right|^{\alpha} \\
& \sim\left|\lambda_{1}^{h}\right|^{\alpha}\left|A_{1}\right|^{\alpha}+\left|C_{1}\right|^{\alpha}-\left|C_{1}-\lambda_{1}^{h} A_{1}\right|^{\alpha}
\end{aligned}
$$

and since

$$
\lim _{x \rightarrow 0} \frac{|a x|^{\alpha}+|c|^{\alpha}-|c-a x|^{\alpha}}{x}=\alpha a c^{\langle\alpha-1\rangle}
$$


for $1<\alpha<2$ and $a, c \in \mathbb{R}$, and $\lambda_{1}^{h} \rightarrow 0$ for $h \rightarrow+\infty$, we obtain

$$
\lim _{h \rightarrow+\infty} \frac{\left|\lambda_{1}^{h} A_{1}\right|^{\alpha}+\left|C_{1}\right|^{\alpha}-\left|C_{1}-\lambda_{1}^{h} A_{1}\right|^{\alpha}}{\lambda_{1}^{h}}=\alpha A_{1} C_{1}^{\langle\alpha-1\rangle}
$$

for a fixed $\mathbf{s}=\left(s_{1}, s_{2}\right) \in S_{2}$. Now since the equalities presented in i) and ii) are true, we obtain

$$
\lim _{h \rightarrow+\infty} \sum_{j=0}^{+\infty} \int_{S_{2}} \frac{\left|\lambda_{1}^{h} A_{1}+\lambda_{2}^{h} B_{1}\right|^{\alpha}+\left|C_{1}\right|^{\alpha}-\left|C_{1}-\left(\lambda_{1}^{h} A_{1}+\lambda_{2}^{h} B_{1}\right)\right|^{\alpha}}{\lambda_{1}^{h}} \Gamma(d s)=\alpha D_{1},
$$

which is equivalent to the fact that

$$
\begin{aligned}
\sum_{j=0}^{+\infty} \int_{S_{2}}\left|\lambda_{1}^{h} A_{1}+\lambda_{2}^{h} B_{1}\right|^{\alpha}+\left|C_{1}\right|^{\alpha}-\left|C_{1}-\left(\lambda_{1}^{h} A_{1}+\lambda_{2}^{h} B_{1}\right)\right|^{\alpha} \Gamma(d s) & \\
& \sim \alpha D_{1} \lambda_{1}^{h} \text { for } h \rightarrow+\infty
\end{aligned}
$$

where

$$
D_{1}:=\sum_{j=0}^{+\infty} \int_{S_{2}} A_{1} C_{1}^{\langle\alpha-1\rangle} \Gamma(d s) .
$$

Let us notice that the conditions given in (27) guarantee that $D_{1}<+\infty$.

II) Consider the case when $\left|\lambda_{1}\right|<\left|\lambda_{2}\right|$. Similarly as above we have

$$
\begin{aligned}
\sum_{j=0}^{+\infty} \int_{S_{2}}\left|\lambda_{1}^{h} A_{1}+\lambda_{2}^{h} B_{1}\right|^{\alpha}+\left|C_{1}\right|^{\alpha}-\left|C_{1}-\left(\lambda_{1}^{h} A_{1}+\lambda_{2}^{h} B_{1}\right)\right|^{\alpha} \Gamma(d s) & \\
& \sim \alpha D_{2} \lambda_{2}^{h} \text { for } h \rightarrow+\infty
\end{aligned}
$$

where

$$
D_{2}:=\sum_{j=0}^{+\infty} \int_{S_{2}} B_{1} C_{1}^{\langle\alpha-1\rangle} \Gamma(d s)<+\infty .
$$

III) Consider the case when $\lambda_{1}=-\lambda_{2}$ and $h$ is even. Proceeding in the same way as above, we obtain

$$
\begin{aligned}
\sum_{j=0}^{+\infty} \int_{S_{2}}\left|\lambda_{1}^{h} A_{1}+\lambda_{2}^{h} B_{1}\right|^{\alpha}+\left|C_{1}\right|^{\alpha}-\mid C_{1}-\left(\lambda_{1}^{h} A_{1}\right. & \left.+\lambda_{2}^{h} B_{1}\right)\left.\right|^{\alpha} \Gamma(d s) \\
& \sim \alpha\left(D_{1}+D_{2}\right) \lambda_{1}^{h} \text { for } h \rightarrow+\infty,
\end{aligned}
$$

where

$$
D_{1}+D_{2}=\sum_{j=0}^{+\infty} \int_{S_{2}}\left(A_{1}+B_{1}\right) C_{1}^{\langle\alpha-1\rangle} \Gamma(d s)<+\infty
$$

IV) Consider the case when $\lambda_{1}=-\lambda_{2}$ and $h$ is odd. Similarly to the previous cases, we have

$$
\begin{aligned}
\sum_{j=0}^{+\infty} \int_{S_{2}}\left|\lambda_{1}^{h} A_{1}+\lambda_{2}^{h} B_{1}\right|^{\alpha}+\left|C_{1}\right|^{\alpha}-\mid C_{1}-\left(\lambda_{1}^{h} A_{1}\right. & \left.+\lambda_{2}^{h} B_{1}\right)\left.\right|^{\alpha} \Gamma(d s) \\
& \sim \alpha\left(D_{1}-D_{2}\right) \lambda_{1}^{h} \text { for } h \rightarrow+\infty
\end{aligned}
$$


where

$$
D_{1}-D_{2}=\sum_{j=0}^{+\infty} \int_{S_{2}}\left(A_{1}-B_{1}\right) C_{1}^{\langle\alpha-1\rangle} \Gamma(d s)<+\infty .
$$

- Consider the case of the real and equal eigenvalues of the coefficient matrix $\Theta$, $\lambda_{1}=\lambda_{2}=\lambda$, and $|\lambda|<1$. For the codifference $\operatorname{CD}\left(X_{1}(t), X_{2}(t-h)\right)$ given in Lemma 3.3 (see 13 ) ) we introduce the notation

$$
\begin{aligned}
& A_{2}\left(s_{1}, s_{2}, a_{1}, a_{2}, \lambda, j\right)=A_{2}=j \lambda^{j-1} a_{1} s_{1}-j \lambda^{j} s_{1}+\lambda^{j} s_{1}+j \lambda^{j-1} a_{2} s_{2}, \\
& B_{2}\left(s_{1}, s_{2}, a_{1}, a_{2}, \lambda, j\right)=B_{2}=\lambda^{j-1} a_{1} s_{2}-\lambda^{j} s_{1}+\lambda^{j-1} a_{2} s_{2}, \\
& C_{2}\left(s_{1}, s_{2}, a_{3}, a_{4}, \lambda, j\right)=C_{2}=j \lambda^{j-1} a_{3} s_{1}+j \lambda^{j-1} a_{4} s_{2}-j \lambda^{j} s_{2}+\lambda^{j} s_{2} .
\end{aligned}
$$

Again, the above expressions will be referred to as $A_{2}, B_{2}$ and $C_{2}$ to simplify the notation. Now, the cross-codifference function $\mathrm{CD}\left(X_{1}(t), X_{2}(t-h)\right)$ given in 13$)$ takes the form

$$
\begin{aligned}
& \operatorname{CD}\left(X_{1}(t), X_{2}(t-h)\right) \\
& \quad=\sum_{j=0}^{+\infty} \int_{S_{2}}\left(\left|\lambda^{h} A_{2}+h \lambda^{h} B_{2}\right|^{\alpha}+\left|C_{2}\right|^{\alpha}-\left|C_{2}-\left(\lambda^{h} A_{2}+h \lambda^{h} B_{2}\right)\right|^{\alpha}\right) \Gamma(d s) .
\end{aligned}
$$

Now, similarly to the case of two different eigenvalues, we split the proof into two parts. Namely, we want to show that

$$
\text { i) } \begin{aligned}
& \lim _{h \rightarrow+\infty} \sum_{j=0}^{+\infty} \int_{S_{2}} \frac{\left|\lambda^{h} A_{2}+h \lambda^{h} B_{2}\right|^{\alpha}+\left|C_{2}\right|^{\alpha}-\left|C_{2}-\left(\lambda^{h} A_{2}+h \lambda^{h} B_{2}\right)\right|^{\alpha}}{h \lambda^{h}} \Gamma(d s) \\
= & \sum_{j=0}^{+\infty} \lim _{h \rightarrow+\infty} \int_{S_{2}} \frac{\left|\lambda^{h} A_{2}+h \lambda^{h} B_{2}\right|^{\alpha}+\left|C_{2}\right|^{\alpha}-\left|C_{2}-\left(\lambda^{h} A_{2}+h \lambda^{h} B_{2}\right)\right|^{\alpha}}{h \lambda^{h}} \Gamma(d s),
\end{aligned}
$$

ii) $\lim _{h \rightarrow+\infty} \int_{S_{2}} \frac{\left|\lambda^{h} A_{2}+h \lambda^{h} B_{2}\right|^{\alpha}+\left|C_{2}\right|^{\alpha}-\left|C_{2}-\left(\lambda^{h} A_{2}+h \lambda^{h} B_{2}\right)\right|^{\alpha}}{h \lambda^{h}} \Gamma(d s)$

$$
=\int_{S_{2}} \lim _{h \rightarrow+\infty} \frac{\left|\lambda^{h} A_{2}+h \lambda^{h} B_{2}\right|^{\alpha}+\left|C_{2}\right|^{\alpha}-\left|C_{2}-\left(\lambda^{h} A_{2}+h \lambda^{h} B_{2}\right)\right|^{\alpha}}{h \lambda^{h}} \Gamma(d s) .
$$

To justify the equality in part i), according to the dominated convergence theorem, the infinite sum over $j$ has to converge uniformly. Using the inequalities given in $(26)$ we can show that for all $j \in \mathbb{N}$ we have

$$
\begin{aligned}
& \left|\int_{S_{2}} \frac{\left|\lambda^{h} A_{2}+h \lambda^{h} B_{2}\right|^{\alpha}+\left|C_{2}\right|^{\alpha}-\left|C_{2}-\left(\lambda^{h} A_{2}+h \lambda^{h} B_{2}\right)\right|^{\alpha}}{h \lambda^{h}} \Gamma(d s)\right| \\
& \leq \int_{S_{2}} \frac{|| \lambda^{h} A_{2}+\left.h \lambda^{h} B_{2}\right|^{\alpha}+\left|C_{2}\right|^{\alpha}-\left|C_{2}-\left(\lambda^{h} A_{2}+h \lambda^{h} B_{2}\right)\right|^{\alpha}}{h \lambda^{h}} \mid \Gamma(d s) \\
& \leq \int_{S_{2}} \frac{(\alpha+1)\left|\lambda^{h} A_{2}+h \lambda^{h} B_{2}\right|^{\alpha}+\alpha\left|\lambda^{h} A_{2}+h \lambda^{h} B_{2}\right|\left|C_{2}\right|^{\alpha-1}}{\left|h \lambda^{h}\right|} \Gamma(d s) \\
& =(\alpha+1)\left|h \lambda^{h}\right|^{\alpha-1} \int_{S_{2}}\left|\frac{A_{2}}{h}+B_{2}\right|^{\alpha} \Gamma(d s)+\alpha \int_{S_{2}}\left|\frac{A_{2}}{h}+B_{2}\right|\left|C_{2}\right|^{\alpha-1} \Gamma(d s)
\end{aligned}
$$




$$
\begin{aligned}
\leq & (\alpha+1)\left|h \lambda^{h}\right|^{\alpha-1} 2^{\alpha-1} \int_{S_{2}}\left(\left|\frac{A_{2}}{h}\right|^{\alpha}+\left|B_{2}\right|^{\alpha}\right) \Gamma(d s)+\alpha \int_{S_{2}}\left(\left|\frac{A_{2}}{h}\right|+\left|B_{2}\right|\right)|C|^{\alpha-1} \Gamma(d s) \\
\leq & 2^{\alpha-1}(\alpha+1) M\left(\int_{S_{2}}\left|A_{2}\right|^{\alpha} \Gamma(d s)+\int_{S_{2}}\left|B_{2}\right|^{\alpha} \Gamma(d s)\right) \\
& +\alpha\left(\int_{S_{2}}\left|A_{2}\right|\left|C_{2}\right|^{\alpha-1} \Gamma(d s)+\int_{S_{2}}\left|B_{2}\right|\left|C_{2}\right|^{\alpha-1} \Gamma(d s)\right)=N_{j},
\end{aligned}
$$

where $M$ denotes the boundary of the sequence $\left\{h \lambda^{h}\right\}$ for $h \in \mathbb{N}$ (the sequence converges). Let us notice that $N_{j}$ is independent of $h$. Now, the infinite sum under the limit in (38) converges uniformly if the infinite sum of $N_{j}$ over $j \in \mathbb{N}$ converges, which is equivalent to the following set of conditions

$$
\begin{array}{ll}
\sum_{j=0}^{+\infty} \int_{S_{2}}\left|A_{2}\right|^{\alpha} \Gamma(d s)<+\infty, & \sum_{j=0}^{+\infty} \int_{S_{2}}\left|A_{2}\right|\left|C_{2}\right|^{\alpha-1} \Gamma(d s)<+\infty, \\
\sum_{j=0}^{+\infty} \int_{S_{2}}\left|B_{2}\right|^{\alpha} \Gamma(d s)<+\infty, & \sum_{j=0}^{+\infty} \int_{S_{2}}\left|B_{2}\right|\left|C_{2}\right|^{\alpha-1} \Gamma(d s)<+\infty .
\end{array}
$$

The above conditions are always satisfied (see Remark C.1) and consequently the equality given in $(38)$ is true.

Let us focus on part ii). Similarly, according to the dominated convergence theorem, the equality given in $(39)$ is satisfied if the integrand is dominated by an integrable function independent of $h$. Let us notice that using the inequalities given in (26) for a fixed $\mathbf{s}=\left(s_{1}, s_{2}\right) \in S_{2}$ we have

$$
\begin{aligned}
& \left|\frac{\left|\lambda^{h} A_{2}+h \lambda^{h} B_{2}\right|^{\alpha}+\left|C_{2}\right|^{\alpha}-\left|C_{2}-\left(\lambda^{h} A_{2}+h \lambda^{h} B_{2}\right)\right|^{\alpha}}{h \lambda^{h}}\right| \\
& \leq \frac{(\alpha+1)\left|\lambda^{h} A_{2}+h \lambda^{h} B_{2}\right|^{\alpha}+\alpha\left|\lambda^{h} A_{2}+h \lambda^{h} B_{2}\right|\left|C_{2}\right|^{\alpha-1}}{\left|h \lambda^{h}\right|} \\
& =(\alpha+1)\left|h \lambda^{h}\right|^{\alpha-1}\left|\frac{A_{2}}{h}+B_{2}\right|^{\alpha}+\alpha\left|\frac{A_{2}}{h}+B_{2}\right||C|^{\alpha-1} \\
& \leq(\alpha+1)\left|h \lambda^{h}\right|^{\alpha-1} 2^{\alpha-1}\left(\left|\frac{A_{2}}{h}\right|^{\alpha}+\left|B_{2}\right|^{\alpha}\right)+\alpha\left(\left|\frac{A_{2}}{h}\right|+\left|B_{2}\right|\right)\left|C_{2}\right|^{\alpha-1} \\
& \leq 2^{\alpha-1}(\alpha+1) M\left(\left|A_{2}\right|^{\alpha}+\left|B_{2}\right|^{\alpha}\right)+\alpha\left(\left|A_{2}\right|\left|C_{2}\right|^{\alpha-1}+\left|B_{2}\right|\left|C_{2}\right|^{\alpha-1}\right),
\end{aligned}
$$

which is independent of $h$. Since the dominating function should be integrable we obtain that for all $j \in \mathbb{N}$ the following set of conditions has to be satisfied

$$
\begin{array}{ll}
\int_{S_{2}}\left|A_{2}\right|^{\alpha} \Gamma(d s)<+\infty, & \int_{S_{2}}\left|A_{2}\right|\left|C_{2}\right|^{\alpha-1} \Gamma(d s)<+\infty, \\
\int_{S_{2}}\left|B_{2}\right|^{\alpha} \Gamma(d s)<+\infty, & \int_{S_{2}}\left|B_{2}\right|\left|C_{2}\right|^{\alpha-1} \Gamma(d s)<+\infty .
\end{array}
$$

Let us notice that the above conditions are satisfied since the conditions given in 40 hold. Now, we calculate the limit of the integrand given in 39 for a fixed $\mathbf{s}=\left(s_{1}, s_{2}\right) \in S_{2}$, namely

$$
\lim _{h \rightarrow+\infty} \frac{\left|\lambda^{h} A_{2}+h \lambda^{h} B_{2}\right|^{\alpha}+\left|C_{2}\right|^{\alpha}-\left|C_{2}-\left(\lambda^{h} A_{2}+h \lambda^{h} B_{2}\right)\right|^{\alpha}}{h \lambda^{h}} .
$$


Let us notice that for $h \rightarrow+\infty$ we have

$$
\begin{aligned}
& \left|\lambda^{h} A_{2}+h \lambda^{h} B_{2}\right|^{\alpha}+\left|C_{2}\right|^{\alpha}-\left|C_{2}-\left(\lambda^{h} A_{2}+h \lambda^{h} B_{2}\right)\right|^{\alpha} \\
& =\left|h \lambda^{h}\left(A_{2} / h+B_{2}\right)\right|^{\alpha}+\left|C_{2}\right|^{\alpha}-\left|C_{2}-h \lambda^{h}\left(A_{2} / h+B_{2}\right)\right|^{\alpha} \\
& \sim\left|h \lambda^{h}\right|^{\alpha}\left|B_{2}\right|^{\alpha}+\left|C_{2}\right|^{\alpha}-\left|C_{2}-h \lambda^{h} B_{2}\right|^{\alpha} .
\end{aligned}
$$

Now, using the limit given in 28 and since $h \lambda^{h} \rightarrow 0$ for $h \rightarrow+\infty$, we obtain

$$
\lim _{h \rightarrow+\infty} \frac{\left|h \lambda^{h} B_{2}\right|^{\alpha}+\left|C_{2}\right|^{\alpha}-\left|C_{2}-h \lambda^{h} B_{2}\right|^{\alpha}}{h \lambda^{h}}=\alpha B_{2} C_{2}^{\langle\alpha-1\rangle}
$$

for a fixed $\mathbf{s}=\left(s_{1}, s_{2}\right) \in S_{2}$. Now since the equalities in i) and ii) are true, we have

$$
\lim _{h \rightarrow+\infty} \sum_{j=0}^{+\infty} \int_{S_{2}} \frac{\left|\lambda^{h} A_{2}+h \lambda^{h} B_{2}\right|^{\alpha}+\left|C_{2}\right|^{\alpha}-\left|C_{2}-\left(\lambda^{h} A_{2}+h \lambda^{h} B_{2}\right)\right|^{\alpha}}{h \lambda^{h}} \Gamma(d s)=\alpha D_{3},
$$

which is equivalent to the fact that

$$
\begin{aligned}
\sum_{j=0}^{+\infty} \int_{S_{2}}\left|\lambda^{h} A_{2}+h \lambda^{h} B_{2}\right|^{\alpha}+\left|C_{2}\right|^{\alpha}-\left|C_{2}-\left(\lambda^{h} A_{2}+h \lambda^{h} B_{2}\right)\right|^{\alpha} \Gamma(d s) & \\
& \sim \alpha D_{3} h \lambda^{h} \text { for } h \rightarrow+\infty
\end{aligned}
$$

where

$$
D_{3}:=\sum_{j=0}^{+\infty} \int_{S_{2}} B_{2} C_{2}^{\langle\alpha-1\rangle} \Gamma(d s) .
$$

Let us notice that the conditions given in 40 guarantee that $D_{3}<+\infty$.

b) Now, we examine the asymptotic behavior of $\operatorname{CD}\left(X_{1}(t), X_{2}(t+h)\right)$ for $h \rightarrow+\infty$.

- Let us consider the case of two different real eigenvalues of the coefficient matrix $\Theta$, $\lambda_{1} \neq \lambda_{2}$, and $\left|\lambda_{1}\right|<1,\left|\lambda_{2}\right|<1$. For the cross-codifference function $\operatorname{CD}\left(X_{1}(t), X_{2}(t+h)\right)$ given in Lemma 3.3 (see 12 ) we introduce the notation

$$
\begin{aligned}
A_{3}\left(s_{1}, s_{2}, a_{3}, a_{4}, \lambda_{1}, \lambda_{2}, j\right) & =A_{3}=\frac{-\lambda_{1}^{j} a_{3} s_{1}+\lambda_{2} \lambda_{1}^{j} s_{2}-\lambda_{1}^{j} a_{4} s_{2}}{\lambda_{2}-\lambda_{1}}, \\
B_{3}\left(s_{1}, s_{2}, a_{3}, a_{4}, \lambda_{1}, \lambda_{2}, j\right) & =B_{3}=\frac{\lambda_{2}^{j} a_{3} s_{1}-\lambda_{1} \lambda_{2}^{j} s_{2}+\lambda_{2}^{j} a_{4} s_{2}}{\lambda_{2}-\lambda_{1}}, \\
C_{3}\left(s_{1}, s_{2}, a_{1}, a_{2}, \lambda_{1}, \lambda_{2}, j\right) & =C_{3} \\
& =\frac{\lambda_{1}^{j}\left(\lambda_{2} s_{1}-a_{1} s_{1}-a_{2} s_{2}\right)+\lambda_{2}^{j}\left(-\lambda_{1} s_{1}+a_{1} s_{1}+a_{2} s_{2}\right)}{\lambda_{2}-\lambda_{1}} .
\end{aligned}
$$

Again, the above expressions will be referred to as $A_{3}, B_{3}$ and $C_{3}$ to simplify the notation. Now, we can write the formula 12 for $\operatorname{CD}\left(X_{1}(t), X_{2}(t+h)\right)$ in the form

$$
\begin{aligned}
\operatorname{CD}\left(X_{1}(t), X_{2}(t+h)\right) & \\
& =\sum_{j=0}^{+\infty} \int_{S_{2}}\left(\left|\lambda_{1}^{h} A_{3}+\lambda_{2}^{h} B_{3}\right|^{\alpha}+\left|C_{3}\right|^{\alpha}-\left|C_{3}-\left(\lambda_{1}^{h} A_{3}+\lambda_{2}^{h} B_{3}\right)\right|^{\alpha}\right) \Gamma(d s) .
\end{aligned}
$$


I) Consider the case of $\left|\lambda_{1}\right|>\left|\lambda_{2}\right|$. Proceeding exactly as in a) we obtain

$$
\begin{aligned}
\sum_{j=0}^{+\infty} \int_{S_{2}}\left|\lambda_{1}^{h} A_{3}+\lambda_{2}^{h} B_{3}\right|^{\alpha}+\left|C_{3}\right|^{\alpha}-\left|C_{3}-\left(\lambda_{1}^{h} A_{3}+\lambda_{2}^{h} B_{3}\right)\right|^{\alpha} \Gamma(d s) & \\
& \sim \alpha D_{4} \lambda_{1}^{h} \text { for } h \rightarrow+\infty,
\end{aligned}
$$

where

$$
D_{4}:=\sum_{j=0}^{+\infty} \int_{S_{2}} A_{3} C_{3}^{\langle\alpha-1\rangle} \Gamma(d s)<+\infty .
$$

Let us mention that to apply the dominated convergence theorem the following conditions have to hold

$$
\begin{array}{ll}
\sum_{j=0}^{+\infty} \int_{S_{2}}\left|A_{3}\right|^{\alpha} \Gamma(d s)<+\infty, & \sum_{j=0}^{+\infty} \int_{S_{2}}\left|A_{3}\right|\left|C_{3}\right|^{\alpha-1} \Gamma(d s)<+\infty, \\
\sum_{j=0}^{+\infty} \int_{S_{2}}\left|B_{3}\right|^{\alpha} \Gamma(d s)<+\infty, & \sum_{j=0}^{+\infty} \int_{S_{2}}\left|B_{3}\right|\left|C_{3}\right|^{\alpha-1} \Gamma(d s)<+\infty,
\end{array}
$$

which is always true (see Remark C.1.).

II) Consider the case of $\left|\lambda_{1}\right|<\left|\lambda_{2}\right|$. Again, proceeding exactly as above we obtain

$$
\begin{aligned}
\sum_{j=0}^{+\infty} \int_{S_{2}}\left|\lambda_{1}^{h} A_{3}+\lambda_{2}^{h} B_{3}\right|^{\alpha}+\left|C_{3}\right|^{\alpha}-\left|C_{3}-\left(\lambda_{1}^{h} A_{3}+\lambda_{2}^{h} B_{3}\right)\right|^{\alpha} \Gamma(d s) & \\
& \sim \alpha D_{5} \lambda_{2}^{h} \text { for } h \rightarrow+\infty,
\end{aligned}
$$

where

$$
D_{5}:=\sum_{j=0}^{+\infty} \int_{S_{2}} B_{3} C_{3}^{\langle\alpha-1\rangle} \Gamma(d s)<+\infty .
$$

III) Consider the case where $\lambda_{1}=-\lambda_{2}$ and $h$ is even. Proceeding as above we have

$$
\begin{aligned}
\sum_{j=0}^{+\infty} \int_{S_{2}}\left|\lambda_{1}^{h} A_{3}+\lambda_{2}^{h} B_{3}\right|^{\alpha}+\left|C_{3}\right|^{\alpha}-\mid C_{3}-\left(\lambda_{1}^{h} A_{3}\right. & \left.+\lambda_{2}^{h} B_{3}\right)\left.\right|^{\alpha} \Gamma(d s) \\
& \sim \alpha\left(D_{4}+D_{5}\right) \lambda_{1}^{h} \text { for } h \rightarrow+\infty,
\end{aligned}
$$

where $D_{4}$ and $D_{5}$ are the constants defined in (47) and (50).

IV) Consider the case where $\lambda_{1}=-\lambda_{2}$ and $h$ is odd. Proceeding as above we have

$$
\begin{aligned}
\sum_{j=0}^{+\infty} \int_{S_{2}}\left|\lambda_{1}^{h} A_{3}+\lambda_{2}^{h} B_{3}\right|^{\alpha}+\left|C_{3}\right|^{\alpha}-\mid C_{3}-\left(\lambda_{1}^{h} A_{3}\right. & \left.+\lambda_{2}^{h} B_{3}\right)\left.\right|^{\alpha} \Gamma(d s) \\
& \sim \alpha\left(D_{4}-D_{5}\right) \lambda_{1}^{h} \text { for } h \rightarrow+\infty,
\end{aligned}
$$

where $D_{4}$ and $D_{5}$ are defined in 47 and $(50)$.

- Consider the case of real and equal eigenvalues of the coefficient matrix $\Theta$, $\lambda_{1}=\lambda_{2}=\lambda$, and $|\lambda|<1$. For the cross-codifference function $\operatorname{CD}\left(X_{1}(t), X_{2}(t+h)\right)$ given 
in Lemma 3.3 (see 14 ) we take the notation

$$
\begin{aligned}
& A_{4}\left(s_{1}, s_{2}, a_{3}, a_{4}, \lambda, j\right)=A_{4}=j \lambda^{j-1} a_{3} s_{1}+j \lambda^{j-1} a_{4} s_{2}-j \lambda^{j} s_{2}+\lambda^{j} s_{2}, \\
& B_{4}\left(s_{1}, s_{2}, a_{1}, a_{2}, \lambda, j\right)=B_{4}=\lambda^{j-1} a_{3} s_{1}+\lambda^{j-1} a_{4} s_{2}-\lambda^{j} s_{2}, \\
& C_{4}\left(s_{1}, s_{2}, a_{3}, a_{4}, \lambda, j\right)=C_{4}=j \lambda^{j-1} a_{1} s_{1}-j \lambda^{j} s_{1}+\lambda^{j} s_{1}+j \lambda^{j-1} a_{2} s_{2} .
\end{aligned}
$$

Referring to the above expressions as $A_{4}, B_{4}$ and $C_{4}$ to simplify the notation, we can write the cross-codifference function $\mathrm{CD}\left(X_{1}(t), X_{2}(t+h)\right)$ given in 14 in the form

$$
\begin{aligned}
& \mathrm{CD}\left(X_{1}(t), X_{2}(t+h)\right) \\
& \quad=\sum_{j=0}^{+\infty} \int_{S_{2}}\left(\left|\lambda^{h} A_{4}+h \lambda^{h} B_{4}\right|^{\alpha}+\left|C_{4}\right|^{\alpha}-\left|C_{4}-\left(\lambda^{h} A_{4}+h \lambda^{h} B_{4}\right)\right|^{\alpha}\right) \Gamma(d s) .
\end{aligned}
$$

Now, proceeding as in a) we obtain

$$
\begin{aligned}
\sum_{j=0}^{+\infty} \int_{S_{2}}\left|\lambda^{h} A_{4}+h \lambda^{h} B_{4}\right|^{\alpha}+\left|C_{4}\right|^{\alpha}-\left|C_{4}-\left(\lambda^{h} A_{4}+h \lambda^{h} B_{4}\right)\right|^{\alpha} \Gamma(d s) & \\
& \sim \alpha D_{6} h \lambda^{h} \text { for } h \rightarrow+\infty
\end{aligned}
$$

where

$$
D_{6}:=\sum_{j=0}^{+\infty} \int_{S_{2}} B_{4} C_{4}^{\langle\alpha-1\rangle} \Gamma(d s)<+\infty .
$$

Similarly as above, to apply the dominated convergence theorem the following conditions have to hold

$$
\begin{aligned}
& \sum_{j=0}^{+\infty} \int_{S_{2}}\left|A_{4}\right|^{\alpha} \Gamma(d s)<+\infty, \quad \sum_{j=0}^{+\infty} \int_{S_{2}}\left|A_{4}\right|\left|C_{4}\right|^{\alpha-1} \Gamma(d s)<+\infty, \\
& \sum_{j=0}^{+\infty} \int_{S_{2}}\left|B_{4}\right|^{\alpha} \Gamma(d s)<+\infty, \quad \sum_{j=0}^{+\infty} \int_{S_{2}}\left|B_{4}\right|\left|C_{4}\right|^{\alpha-1} \Gamma(d s)<+\infty,
\end{aligned}
$$

which is always satisfied (see Remark C.1).

\section{Appendix B: Proof of Lemma 4.2}

a) First, we examine the asymptotic behavior of $\mathrm{CV}\left(X_{1}(t), X_{2}(t-h)\right)$ for $h \rightarrow+\infty$.

- Consider the case of two different real eigenvalues of the coefficient matrix $\Theta$, $\lambda_{1} \neq \lambda_{2}$, and $\left|\lambda_{1}\right|<1,\left|\lambda_{2}\right|<1$. By using the notation introduced in 22 the crosscovariation function $\mathrm{CV}\left(X_{1}(t), X_{2}(t-h)\right)$ given in Lemma 3.4 (see (15) can be written in the form

$$
\mathrm{CV}\left(X_{1}(t), X_{2}(t-h)\right)=\lambda_{1}^{h} D_{1}+\lambda_{2}^{h} D_{2},
$$

where $D_{1}$ and $D_{2}$ are specified in 31 and $(33)$, respectively. The formula given in 58 directly leads to the expressions given in Lemma 4.2 .

- Consider the case of the real and equal eigenvalues of the coefficient matrix $\Theta$, $\lambda_{1}=\lambda_{2}=\lambda$, and $|\lambda|<1$. By using the notation introduced in 36 the cross-covariation 
function $\mathrm{CV}\left(X_{1}(t), X_{2}(t-h)\right)$ given in Lemma 3.4 (see (17)) can be written in the following form

$$
\mathrm{CV}\left(X_{1}(t), X_{2}(t-h)\right)=\lambda^{h} E_{3}+h \lambda^{h} D_{3},
$$

where $D_{3}$ is given in 43 and

$$
E_{3}=\sum_{j=0}^{+\infty} \int_{S_{2}} A_{2} C_{2}^{\langle\alpha-1\rangle} \Gamma(d s) .
$$

Similarly to the previous case, the formula given in $(59)$ directly leads to the expression given in Lemma 4.2

b) Now, we examine the asymptotic behavior of $\mathrm{CV}\left(X_{1}(t), X_{2}(t+h)\right)$ for $h \rightarrow+\infty$.

- Let us consider the case of two different real eigenvalues of the coefficient matrix $\Theta$, $\lambda_{1} \neq \lambda_{2}$, and $\left|\lambda_{1}\right|<1,\left|\lambda_{2}\right|<1$. Taking the notation introduced in (44) the crosscovariation function $\mathrm{CV}\left(X_{1}(t), X_{2}(t+h)\right)$ given in Lemma 3.4 (see (16)) can be written in the form

$$
\mathrm{CV}\left(X_{1}(t), X_{2}(t+h)\right)=\sum_{j=0}^{+\infty} \int_{S_{2}} C_{3}\left(A_{3} \lambda_{1}^{h}+B_{3} \lambda_{2}^{h}\right)^{\langle\alpha-1\rangle} \Gamma(d s) .
$$

I) Let us consider the case of $\left|\lambda_{1}\right|>\left|\lambda_{2}\right|$. Now, similarly as in Lemma 4.1, we split the proof into two parts. Namely, we show that

$$
\text { i) } \begin{aligned}
\lim _{h \rightarrow+\infty} \sum_{j=0}^{+\infty} \int_{S_{2}} \frac{C_{3}\left(A_{3} \lambda_{1}^{h}+B_{3} \lambda_{2}^{h}\right)^{\langle\alpha-1\rangle}}{\left(\lambda_{1}^{h}\right)^{\langle\alpha-1\rangle}} \Gamma(d s) \\
=\sum_{j=0}^{+\infty} \lim _{h \rightarrow+\infty} \int_{S_{2}} \frac{C_{3}\left(A_{3} \lambda_{1}^{h}+B_{3} \lambda_{2}^{h}\right)^{\langle\alpha-1\rangle}}{\left(\lambda_{1}^{h}\right)^{\langle\alpha-1\rangle}} \Gamma(d s),
\end{aligned}
$$

ii) $\lim _{h \rightarrow+\infty} \int_{S_{2}} \frac{C_{3}\left(A_{3} \lambda_{1}^{h}+B_{3} \lambda_{2}^{h}\right)^{\langle\alpha-1\rangle}}{\left(\lambda_{1}^{h}\right)^{\langle\alpha-1\rangle}} \Gamma(d s)$

$$
=\int_{S_{2}} \lim _{h \rightarrow+\infty} \frac{C_{3}\left(A_{3} \lambda_{1}^{h}+B_{3} \lambda_{2}^{h}\right)^{\langle\alpha-1\rangle}}{\left(\lambda_{1}^{h}\right)^{\langle\alpha-1\rangle}} \Gamma(d s) .
$$

At first, let us focus on part i). According to the dominated convergence theorem, the equality given in 61 is true if the infinite sum over $j$ converges uniformly. Let us notice that using the following inequality true for all $n, m \in \mathbb{R}, 1<\alpha<2$

$$
|n+m|^{\alpha-1} \leq|n|^{\alpha-1}+|m|^{\alpha-1}
$$

one can show that for all $j \in \mathbb{N}$ we have

$$
\begin{aligned}
& \left|\int_{S_{2}} \frac{C_{3}\left(A_{3} \lambda_{1}^{h}+B_{3} \lambda_{2}^{h}\right)^{\langle\alpha-1\rangle}}{\left(\lambda_{1}^{h}\right)^{\langle\alpha-1\rangle}} \Gamma(d s)\right| \leq \int_{S_{2}} \frac{\mid C_{3}\left(A_{3} \lambda_{1}^{h}+B_{3} \lambda_{2}^{h}\right)^{\langle\alpha-1\rangle}}{\left(\lambda_{1}^{h}\right)^{\langle\alpha-1\rangle}} \mid \Gamma(d s) \\
& \leq \int_{S_{2}} \frac{\left|C_{3}\right|\left|A_{3} \lambda_{1}^{h}+B_{3} \lambda_{2}^{h}\right|^{\alpha-1}}{\left|\lambda_{1}^{h}\right|^{\alpha-1}} \Gamma(d s)=\int_{S_{2}} \frac{\left|C_{3}\right|\left|\lambda_{1}^{h}\right|^{\alpha-1}\left|A_{3}+B_{3}\left(\lambda_{2} / \lambda_{1}\right)^{h}\right|^{\alpha-1}}{\left|\lambda_{1}^{h}\right|^{\alpha-1}} \Gamma(d s) \\
& =\int_{S_{2}}\left|C_{3}\right|\left|A_{3}+B_{3}\left(\lambda_{2} / \lambda_{1}\right)^{h}\right|^{\alpha-1} \Gamma(d s)
\end{aligned}
$$




$$
\begin{aligned}
& \leq \int_{S_{2}}\left|C_{3}\right|\left(\left|A_{3}\right|^{\alpha-1}+\left|B_{3}\right|^{\alpha-1}\left|\left(\lambda_{2} / \lambda_{1}\right)^{h}\right|^{\alpha-1}\right) \Gamma(d s) \\
& \leq \int_{S_{2}}\left|C_{3}\right|\left|A_{3}\right|^{\alpha-1} \Gamma(d s)+\int_{S_{2}}\left|C_{3}\right|\left|B_{3}\right|^{\alpha-1} \Gamma(d s)=K_{j}
\end{aligned}
$$

and $K_{j}$ is independent of $h$. Now, the infinite sum under the limit in 61 converges uniformly if the infinite sum of $M_{j}$ over $j \in \mathbb{N}$ converges, which is equivalent to the following conditions

$$
\sum_{j=0}^{+\infty} \int_{S_{2}}\left|C_{3}\right|\left|A_{3}\right|^{\alpha-1} \Gamma(d s)<+\infty, \quad \sum_{j=0}^{+\infty} \int_{S_{2}}\left|C_{3}\right|\left|B_{3}\right|^{\alpha-1} \Gamma(d s)<+\infty,
$$

which are always satisfied (see Remark C.1 and thus the equality given in 61) is true. Let us focus on part ii). According to the dominated convergence theorem, the equality given in 62 is satisfied if the integrand is dominated by an integrable function independent of $h$. Using the formula given in 63 for a fixed $\mathbf{s}=\left(s_{1}, s_{2}\right) \in S_{2}$ we obtain

$$
\begin{aligned}
& \left|\frac{C_{3}\left(A_{3} \lambda_{1}^{h}+B_{3} \lambda_{2}^{h}\right)^{\langle\alpha-1\rangle}}{\left(\lambda_{1}^{h}\right)^{\langle\alpha-1\rangle}}\right| \leq \frac{\left|C_{3}\right|\left|A_{3} \lambda_{1}^{h}+B_{3} \lambda_{2}^{h}\right|^{\alpha-1}}{\left|\lambda_{1}^{h}\right|^{\alpha-1}} \\
& =\frac{\left|C_{3}\right|\left|\lambda_{1}^{h}\right|^{\alpha-1}\left|A_{3}+B_{3}\left(\lambda_{2} / \lambda_{1}\right)^{h}\right|^{\alpha-1}}{\left|\lambda_{1}^{h}\right|^{\alpha-1}} \leq\left|C_{3}\right|\left(\left|A_{3}\right|^{\alpha-1}+\left|B_{3}\right|^{\alpha-1}\left|\left(\lambda_{2} / \lambda_{1}\right)^{h}\right|^{\alpha-1}\right) \\
& \leq\left|C_{3}\right|\left|A_{3}\right|^{\alpha-1}+\left|C_{3}\right|\left|B_{3}\right|^{\alpha-1}
\end{aligned}
$$

which is independent of $h$. Now, since the dominating function should be integrable we obtain the following conditions for all $j \in \mathbb{N}$

$$
\int_{S_{2}}\left|C_{3}\right|\left|A_{3}\right|^{\alpha-1} \Gamma(d s)<+\infty, \quad \int_{S_{2}}\left|C_{3}\right|\left|B_{3}\right|^{\alpha-1} \Gamma(d s)<+\infty .
$$

Let us notice that the above conditions are satisfied since the conditions given in (64) hold. Now, we calculate the limit of the integrand given in 62 for a fixed $\mathbf{s}=\left(s_{1}, s_{2}\right) \in S_{2}$, namely

$$
\lim _{h \rightarrow+\infty} \frac{C_{3}\left(A_{3} \lambda_{1}^{h}+B_{3} \lambda_{2}^{h}\right)^{\langle\alpha-1\rangle}}{\left(\lambda_{1}^{h}\right)^{\langle\alpha-1\rangle}}=\lim _{h \rightarrow+\infty} C_{3}\left(A_{3}+B_{3}\left(\lambda_{2} / \lambda_{1}\right)^{h}\right)^{\langle\alpha-1\rangle}=C_{3} A_{3}^{\langle\alpha-1\rangle} .
$$

Now, since the equalities presented in i) and ii) are true, we obtain

$$
\lim _{h \rightarrow+\infty} \sum_{j=0}^{+\infty} \int_{S_{2}} \frac{C_{3}\left(A_{3} \lambda_{1}^{h}+B_{3} \lambda_{2}^{h}\right)^{\langle\alpha-1\rangle}}{\left(\lambda_{1}^{h}\right)^{\langle\alpha-1\rangle}} \Gamma(d s)=D_{7} .
$$

which is equivalent to the fact that

$$
\sum_{j=0}^{+\infty} \int_{S_{2}} C_{3}\left(A_{3} \lambda_{1}^{h}+B_{3} \lambda_{2}^{h}\right)^{\langle\alpha-1\rangle} \Gamma(d s) \sim D_{7}\left(\lambda_{1}^{h}\right)^{\langle\alpha-1\rangle} \text { for } h \rightarrow+\infty,
$$

where

$$
D_{7}:=\sum_{j=0}^{+\infty} \int_{S_{2}} C_{3} A_{3}^{\langle\alpha-1\rangle} \Gamma(d s) .
$$

Let us notice that the conditions given in 64 guarantee that $D_{7}<+\infty$. 
II) Consider the case of $\left|\lambda_{1}\right|<\left|\lambda_{2}\right|$. Proceeding in the same way as above, we have

$$
\sum_{j=0}^{+\infty} \int_{S_{2}} C_{3}\left(A_{3} \lambda_{1}^{h}+B_{3} \lambda_{2}^{h}\right)^{\langle\alpha-1\rangle} \Gamma(d s) \sim D_{8}\left(\lambda_{2}^{h}\right)^{\langle\alpha-1\rangle} \text { for } h \rightarrow+\infty,
$$

where

$$
D_{8}:=\sum_{j=0}^{+\infty} \int_{S_{2}} C_{3} B_{3}^{\langle\alpha-1\rangle} \Gamma(d s)<+\infty .
$$

III) Consider $\lambda_{1}=-\lambda_{2}$ and even $h$. In this case we obtain

$$
\sum_{j=0}^{+\infty} \int_{S_{2}} C_{3}\left(A_{3} \lambda_{1}^{h}+B_{3} \lambda_{2}^{h}\right)^{\langle\alpha-1\rangle} \Gamma(d s)=D_{10}\left(\lambda_{1}^{h}\right)^{\langle\alpha-1\rangle},
$$

where

$$
D_{10}=\sum_{j=0}^{+\infty} \int_{S_{2}} C_{3}\left(A_{3}+B_{3}\right)^{\langle\alpha-1\rangle} \Gamma(d s)<+\infty .
$$

IV) Consider $\lambda_{1}=-\lambda_{2}$ and odd $h$. Similarly to the previous case, we have

$$
\sum_{j=0}^{+\infty} \int_{S_{2}} C_{3}\left(A_{3} \lambda_{1}^{h}+B_{3} \lambda_{2}^{h}\right)^{\langle\alpha-1\rangle} \Gamma(d s)=D_{11}\left(\lambda_{1}^{h}\right)^{\langle\alpha-1\rangle},
$$

where

$$
D_{11}=\sum_{j=0}^{+\infty} \int_{S_{2}} C_{3}\left(A_{3}-B_{3}\right)^{\langle\alpha-1\rangle} \Gamma(d s) .
$$

- Consider the case of the real and equal eigenvalues of the coefficient matrix $\Theta$, $\lambda_{1}=\lambda_{2}=\lambda$, and $|\lambda|<1$. Using the notation introduced in (53) the cross-covariation function $\mathrm{CV}\left(X_{1}(t), X_{2}(t+h)\right)$ given in Lemma 3.4 (see $[18)$ can be expressed as follows

$$
\mathrm{CV}\left(X_{1}(t), X_{2}(t+h)\right)=\sum_{j=0}^{+\infty} \int_{S_{2}} C_{4}\left(A_{4} \lambda^{h}+B_{4} h \lambda^{h}\right)^{\langle\alpha-1\rangle} \Gamma(d s) .
$$

Now, similarly to the previous case we split the proof into two parts, namely we show that

i) $\lim _{h \rightarrow+\infty} \sum_{j=0}^{+\infty} \int_{S_{2}} \frac{C_{4}\left(A_{4} \lambda^{h}+B_{4} h \lambda^{h}\right)^{\langle\alpha-1\rangle}}{\left(h \lambda^{h}\right)^{\langle\alpha-1\rangle}} \Gamma(d s)$

$$
=\sum_{j=0}^{+\infty} \lim _{h \rightarrow+\infty} \int_{S_{2}} \frac{C_{4}\left(A_{4} \lambda^{h}+B_{4} h \lambda^{h}\right)^{\langle\alpha-1\rangle}}{\left(h \lambda^{h}\right)^{\langle\alpha-1\rangle}} \Gamma(d s),
$$

ii) $\lim _{h \rightarrow+\infty} \int_{S_{2}} \frac{C_{4}\left(A_{4} \lambda^{h}+B_{4} h \lambda^{h}\right)^{\langle\alpha-1\rangle}}{\left(h \lambda^{h}\right)^{\langle\alpha-1\rangle}} \Gamma(d s)$

$$
=\int_{S_{2}} \lim _{h \rightarrow+\infty} \frac{C_{4}\left(A_{4} \lambda^{h}+B_{4} h \lambda^{h}\right)^{\langle\alpha-1\rangle}}{\left(h \lambda^{h}\right)^{\langle\alpha-1\rangle}} \Gamma(d s) .
$$


Now, according to the dominated convergence theorem, the equality corresponding to the part i), given in (75), is satisfied if the infinite sum over $j \in \mathbb{N}$ converges uniformly. Using inequality $(63)$ one can show that for all $j \in \mathbb{N}$

$$
\begin{aligned}
& \left|\int_{S_{2}} \frac{C_{4}\left(A_{4} \lambda^{h}+B_{4} h \lambda^{h}\right)^{\langle\alpha-1\rangle}}{\left(h \lambda^{h}\right)^{\langle\alpha-1\rangle}} \Gamma(d s)\right| \leq \int_{S_{2}}\left|\frac{C_{4}\left(A_{4} \lambda^{h}+B_{4} h \lambda^{h}\right)^{\langle\alpha-1\rangle}}{\left(h \lambda^{h}\right)^{\langle\alpha-1\rangle}}\right| \Gamma(d s) \\
& \leq \int_{S_{2}} \frac{\left|C_{4}\right|\left|A_{4} \lambda^{h}+B_{4} h \lambda^{h}\right|^{\alpha-1}}{\left|h \lambda^{h}\right|^{\alpha-1}} \Gamma(d s)=\int_{S_{2}} \frac{\left|C_{4}\right|\left|h \lambda^{h}\right|^{\alpha-1}\left|A_{4} / h+B_{4}\right|^{\alpha-1}}{\left|h \lambda^{h}\right|^{\alpha-1}} \Gamma(d s) \\
& =\int_{S_{2}}\left|C_{4}\right|\left|A_{4} / h+B_{4}\right|^{\alpha-1} \Gamma(d s) \leq \int_{S_{2}}\left|C_{4}\right|\left(\left|A_{4} / h\right|^{\alpha-1}+\left|B_{4}\right|^{\alpha-1}\right) \Gamma(d s) \\
& \leq \int_{S_{2}}\left|C_{4}\right|\left|A_{4}\right|^{\alpha-1} \Gamma(d s)+\int_{S_{2}}\left|C_{4}\right|\left|B_{4}\right|^{\alpha-1} \Gamma(d s)=L_{j}
\end{aligned}
$$

and $L_{j}$ is independent of $h$. Therefore, the infinite sum under the limit in 75 converges uniformly if the infinite sum of $L_{j}$ over $j \in \mathbb{N}$ converges, which is equivalent to the following conditions

$$
\sum_{j=0}^{+\infty} \int_{S_{2}}\left|C_{4}\right|\left|A_{4}\right|^{\alpha-1} \Gamma(d s)<+\infty, \quad \sum_{j=0}^{+\infty} \int_{S_{2}}\left|C_{4}\right|\left|B_{4}\right|^{\alpha-1} \Gamma(d s)<+\infty,
$$

which are always satisfied (see Remark C.1. Now, since the equality given in 75 is true, we consider part ii). Similarly, according to the dominated convergence theorem, the equality given in 76 is satisfied if the integrand is dominated by an integrable function independent of $h$. Again, by using the formula given in 63 for a fixed $\mathbf{s}=\left(s_{1}, s_{2}\right) \in S_{2}$ we have

$$
\begin{aligned}
& \mid \frac{C_{4}\left(A_{4} \lambda^{h}+B_{4} h \lambda^{h}\right)^{\langle\alpha-1\rangle}}{\left(h \lambda^{h}\right)^{\langle\alpha-1\rangle} \mid \leq \frac{\left|C_{4}\right|\left|A_{4} \lambda^{h}+B_{4} h \lambda^{h}\right|^{\alpha-1}}{\left|h \lambda^{h}\right|^{\alpha-1}}} \\
& \quad=\frac{\left|C_{4}\right|\left|h \lambda^{h}\right|^{\alpha-1}\left|A_{4} / h+B_{4}\right|^{\alpha-1}}{\left|h \lambda^{h}\right|^{\alpha-1}} \leq\left|C_{4}\right|\left(\left|A_{4} / h\right|^{\alpha-1}+\left|B_{4}\right|^{\alpha-1}\right) \\
& \quad \leq\left|C_{4}\right|\left|A_{4}\right|^{\alpha-1}+\left|C_{4}\right|\left|B_{4}\right|^{\alpha-1},
\end{aligned}
$$

which is independent of $h$. Now, the fact that the dominating function should be integrable leads to the following conditions for all $j \in \mathbb{N}$

$$
\int_{S_{2}}\left|C_{4}\right|\left|A_{4}\right|^{\alpha-1} \Gamma(d s)<+\infty, \quad \int_{S_{2}}\left|C_{4}\right|\left|B_{4}\right|^{\alpha-1} \Gamma(d s)<+\infty,
$$

which are satisfied since conditions 77 hold. Now, we calculate the limit of the integrand given in 76 for a fixed $\mathbf{s}=\left(s_{1}, s_{2}\right) \in S_{2}$, namely

$$
\lim _{h \rightarrow+\infty} \frac{C_{4}\left(A_{4} \lambda^{h}+B_{4} h \lambda^{h}\right)^{\langle\alpha-1\rangle}}{\left(h \lambda^{h}\right)^{\langle\alpha-1\rangle}}=\lim _{h \rightarrow+\infty} C_{4}\left(A_{4} / h+B_{4}\right)^{\langle\alpha-1\rangle}=C_{4} B_{4}^{\langle\alpha-1\rangle} .
$$

Finally, since the equalities presented in i) and ii) are true, we have

$$
\lim _{h \rightarrow+\infty} \sum_{j=0}^{+\infty} \int_{S_{2}} \frac{C_{4}\left(A_{4} \lambda^{h}+B_{4} h \lambda^{h}\right)^{\langle\alpha-1\rangle}}{\left(h \lambda^{h}\right)^{\langle\alpha-1\rangle}} \Gamma(d s)=D_{9}
$$


which is equivalent to the fact that

$$
\sum_{j=0}^{+\infty} \int_{S_{2}} C_{4}\left(A_{4} \lambda^{h}+B_{4} h \lambda^{h}\right)^{\langle\alpha-1\rangle} \Gamma(d s) \sim D_{9}\left(h \lambda^{h}\right)^{\langle\alpha-1\rangle} \text { for } h \rightarrow+\infty,
$$

where

$$
D_{9}:=\sum_{j=0}^{+\infty} \int_{S_{2}} C_{4} B_{4}^{\langle\alpha-1\rangle} \Gamma(d s) .
$$

Let us notice that conditions 77 guarantee that $D_{9}<+\infty$.

\section{Appendix C}

REMARK C.1. Let us notice that since the moduli of $A_{i}, B_{i}, C_{i}$ for $i=1,2,3,4$ can be bounded above either by $M \max \left(\left|\lambda_{1}\right|,\left|\lambda_{2}\right|\right)^{j}$ or by $M \max j\left(\left|\lambda_{1}\right|,\left|\lambda_{2}\right|\right)^{j}$, where the constant $M$ is independent of $j$ and $\max \left(\left|\lambda_{1}\right|,\left|\lambda_{2}\right|\right)<1$, and since the spectral measure is finite, i.e. $\Gamma\left(S_{2}\right)<+\infty$, all conditions given in the proofs of Lemmas 4.1 and 4.2 see (27), 48, (64), 40, (57) and (77), are satisfied for any spectral measure $\Gamma(\cdot)$.

Acknowledgments. We would like to acknowledge the support of the National Center of Science Opus Grant No. 2016/21/B/ST1/00929 Anomalous diffusion processes and their applications in real data modelling.

\section{References}

[1] P. L. Anderson, M. M. Meerschaert, Modeling river flows with heavy tails, Water Resources Research 34 (1998), 2271-2280.

[2] K. Burnecki, A. Wyłomańska, A. Chechkin, Discriminating between light- and heavy-tailed distributions with limit theorem, PLOS ONE 10 (2015), art. e0145604.

[3] K. C. Butler, K. Okada, The relative contribution of conditional mean and volatility in bivariate returns to international stock market indices, Applied Financial Economics 19 (2009), 1-15.

[4] Z. Chen, X. Geng, F. Yin, A harmonic suppression method based on fractional lower order statistics for power system, IEEE Trans. Industrial Electronics 63 (2016), 3745-3755.

[5] J. Damarackas, V. Paulauskas, Spectral covariance and limit theorems for random fields with infinite variance, J. Multivariate Anal. 153 (2016), 156-175.

[6] C. M. Gallagher, A method for fitting stable autoregressive models using the autocovariation function, Statist. Probab. Lett. 53 (2001), 381-390.

[7] L. Gardes, S. Girard, Conditional extremes from heavy-tailed distributions: an application to the estimation of extreme rainfall return levels, Extremes 13 (2010), 177-204.

[8] P. Glasserman, Ph. Heidelberger, P. Shahabuddin, Portfolio value-at-risk with heavy-tailed risk factors, Math. Finance 12 (2002), 239-269.

[9] A. Grzesiek, M. Teuerle, A. Wyłomańska, Cross-codifference for bidimensional VAR(1) time series with infinite variance, Comm. Statist. Simulation Comput., online (October 2019), DOI: 10.1080/03610918.2019.1670840.

[10] A. Grzesiek, G. Sikora, M. Teuerle, A. Wyłomańska, Spatio-temporal dependence measures for bivariate AR(1) models with $\alpha$-stable noise, J. Time Series Anal. 41 (2020), 454-475. 
[11] M. Jabłońska-Sabuka, M. Teuerle, A. Wyłomańska, Bivariate sub-Gaussian model for stock index returns, Phys. A 486 (2017), 628-637.

[12] T. J. Kozubowski, A. K. Panorska, Multivariate geometric stable distributions in financial applications, Math. Comput. Modelling 29 (1999), 83-92.

[13] T. J. Kozubowski, A. K. Panorska, S. T. Rachev, Statistical issues in modeling multivariate stable portfolios, in: Handbook of Heavy Tailed Distributions in Finance, Handbooks in Finance 1, North-Holland, Amsterdam, 2003, 131-167.

[14] J. Kyselý, Coverage probability of bootstrap confidence intervals in heavy-tailed frequency models, with application to precipitation data, Theoret. Appl. Climatology 101 (2010), 345361.

[15] K.-S. Lii, M. Rosenblatt, An approximate maximum likelihood estimation for non-Gaussian non-minimum phase moving average processes, J. Multivariate Anal. 43 (1992), 272-299.

[16] X. Ma, C. L. Nikias, Joint estimation of time delay and frequency delay in impulsive noise using fractional lower order statistics, IEEE Trans. Signal Processing 44 (1996), 2669-2687.

[17] M. Maejima, K. Yamamoto, Long-memory stable Ornstein-Uhlenbeck processes, Electron. J. Probab. 8 (2003), paper no. 19.

[18] S. Mittnik, S. T. Rachev, Alternative multivariate stable distributions and their applications to financial modeling, in: Stable Processes and Related Topics, Progr. Probab. 25, Birkhäuser, Boston, 1991, 107-119.

[19] S. Mittnik, S. T. Rachev, Stable Paretian Models in Finance, Wiley, New York, 2000.

[20] J. P. Nolan, A. K. Panorska, Data analysis for heavy tailed multivariate samples, Comm. Statist. Stochastic Models 13 (1997), 687-702.

[21] J. Nowicka, Asymptotic behavior of the covariation and the codifference for ARMA models with stable innovations, Comm. Statist. Stochastic Models 13 (1997), 673-685.

[22] J. Nowicka, A. Weron, Measures of dependence for ARMA models with stable innovations, Ann. Univ. Mariae Curie-Skłodowska Sect. A 51 (1997), 133-144.

[23] J. Nowicka-Zagrajek, A. Wyłomańska, The dependence structure for PARMA models with $\alpha$-stable innovations, Acta Phys. Polon. B 37 (2006), 3071-3081.

[24] J. Nowicka-Zagrajek, A. Wyłomańska, Measures of dependence for stable AR(1) models with time-varying coefficients, Stoch. Models 24 (2008), 58-70.

[25] J. Obuchowski, A. Wyłomańska, The Ornstein-Uhlenbeck process with non-Gaussian structure, Acta Phys. Polon. B 44 (2013), 1123-1136.

[26] S. J. Press, Multivariate stable distributions, J. Multivariate Anal. 2 (1972), 444-462.

[27] D. Rosadi, Testing for independence in heavy-tailed time series using the codifference function, Comput. Statist. Data Anal. 53 (2009), 4516-4529.

[28] D. Rosadi, Measuring dependence of random variables with finite and infinite variance using the codifference and the generalized codifference function, in: Advances of Science and Technology for Society, AIP Conference Proceedings 1755, Publ. Amer. Inst. Physics, Melville, NY, 2016, art. 120004.

[29] D. Rosadi, M. Deistler, Estimating the codifference function of linear time series models with infinite variance, Metrika 73 (2011), 395-429.

[30] A. Saichev, D. Sornette, Anomalous power law distribution of total lifetimes of branching processes: Application to earthquake aftershock sequences, Phys. Rev. E 70 (2004), art. 046123.

[31] G. Samorodnitsky, M. S. Taqqu, Stable Non-Gaussian Random Processes: Stochastic Models with Infinite Variance, Chapman \& Hall, New York, 1994. 
[32] M. Shao, C. L. Nikias, Signal processing with fractional lower order moments: stable processes and their applications, Proc. IEEE 81 (1993), 986-1010.

[33] S. V. Stoyanov, G. Samorodnitsky, S. Rachev, S. Ortobelli, Computing the portfolio conditional Value-at-Risk in the alpha-stable case, Probab. Math. Statistics 26 (2006), 1-22.

[34] A. J. Weir, Lebesgue Integration and Measure, Cambridge Univ. Press, Cambridge, 1973.

[35] K. S. Williams, The $n$-th power of a $2 \times 2$ matrix, Math. Mag. 65 (1992), 336 .

[36] A. Wyłomańska, A. Chechkin, J. Gajda, I. M. Sokolov, Codifference as a practical tool to measure interdependence, Phys. A 421 (2015), 412-429.

[37] G. Żak, J. Obuchowski, A. Wyłomańska, R. Zimroz, Application of ARMA modelling and alpha-stable distribution for local damage detection in bearings, Diagnostyka 15 (2014), no. $3,3-10$.

[38] G. Żak, A. Wyłomańska, R. Zimroz, Data driven iterative vibration signal enhancement strategy using alpha-stable distribution, Shock and Vibration 2017, art. 3698370.

[39] V. M. Zolotarev, One-dimensional Stable Distributions, Transl. Math. Monogr. 65, Amer. Math. Soc., Providence, 1986. 
